1 Erythrocyte CD55 facilitates the internalization of Plasmodium falciparum

\title{
2 parasites
}

4 Bikash Shakya ${ }^{1}$, Saurabh D. Patel ${ }^{2}$, Yoshihiko Tani ${ }^{3}$, and Elizabeth S. Egan ${ }^{1 *}$

$6 \quad{ }^{1}$ Departments of Pediatrics and Microbiology \& Immunology, Stanford University School

7 of Medicine, Stanford, CA 94305, USA

$8 \quad$ ZZuckerman Institute, Columbia University, New York City, NY 10027, USA

9 3Japanese Red Cross Osaka Blood Center, Osaka, Japan

$11{ }^{*}$ Correspondence: eegan@stanford.edu

\section{Abstract}

14 Invasion of human erythrocytes by the malaria parasite Plasmodium falciparum is a multi-

15 step process. Previously, a forward genetic screen for $P$. falciparum host factors

16 identified erythrocyte CD55 as essential for invasion, but its specific role and how it

17 interfaces with the other factors that mediate this complex process are unknown. Using

18 CRISPR-Cas9 editing, antibody-based inhibition, and live cell imaging, here we show

19 that CD55 is specifically required for parasite internalization. Pre-invasion kinetics,

20 erythrocyte deformability, and echinocytosis were not influenced by CD55, but entry was

21 inhibited when CD55 was blocked or absent. Visualization of parasites attached to CD55-

22 null erythrocytes point to a role for CD55 in progression of the moving junction. Our

23 findings demonstrate that CD55 acts after discharge of the parasite's rhoptry organelles, 
24 and plays a unique role relative to all other invasion receptors. As the requirement for

25 CD55 is strain-transcendent, these results suggest that CD55 or its interacting partners

26 may hold potential as therapeutic targets for malaria.

Key Words

Malaria, CD55, erythrocyte, invasion, Plasmodium falciparum

\section{Introduction}

32 Malaria is caused by Apicomplexan parasites of the genus Plasmodium, of which

33 Plasmodium falciparum is responsible for the majority of severe disease cases in

34 humans. One of the world's major public health problems, malaria causes an estimated

35216 million infections and $\sim 445,000$ deaths annually, primarily among young children and

36 pregnant women (WHO, 2018). P. falciparum has a complex life cycle involving stages in

37 the human and mosquito, but disease only occurs during the blood stage, when parasites

38 infect and replicate in human red blood cells (RBCs). As $P$. falciparum is an obligate

39 intracellular parasite, understanding the molecular determinants of its developmental

40 cycle within RBCs may lead to new therapies. For example, a number of Plasmodium

41 proteins that play key roles during erythrocyte invasion have shown promise as vaccine

42 candidates (Ord, Rodriguez, \& Lobo, 2015; Sack, Kappe, \& Sather, 2017; Salinas, Tang,

43 \& Tolia, 2019). Since natural genetic variation in red cells can influence innate

44 susceptibility to malaria, host erythrocyte factors may also hold potential as therapeutic

45 targets (Taylor \& Fairhurst, 2014). The identification and study of such factors, however, 
46 has been severely limited by the intractability of mature RBCs, which lack a nucleus and

47 DNA.

49 P. falciparum invasion of erythrocytes involves a series of coordinated events that unfold 50 rapidly over the course of $\sim 2$ minutes. These events can be divided into three phases:

51 pre-invasion, active invasion, and echinocytosis (Gilson \& Crabb, 2009; Weiss et al.,

52 2015). The process is initiated with the rupture of a "mother" parasite, termed a schizont,

53 which releases up to 32 daughter merozoites. During the pre-invasion phase, a free

54 merozoite makes initial contact with the red cell, stimulating shallow deformation of the

55 host cell plasma membrane. Next, the merozoite reorients so its apically-localized

56 organelles are abutting the cell surface. Reorientation is associated with significant

57 membrane deformation and involves interactions between $P$. falciparum ligands such as

58 the erythrocyte binding antigen (EBA) and reticulocyte binding-like homologues (Rh)

59 family proteins, and receptors on the red cell surface (Gilson \& Crabb, 2009; Paul, Egan,

60 \& Duraisingh, 2015; Riglar et al., 2011; Tham, Healer, \& Cowman, 2012; Weiss et al.,

61 2015). Several ligand and receptor pairs have been shown to act at this stage, often in a

62 strain-specific manner (e.g. PfEBA-175 and GYPA; PfEBA-181 and GYPB PfEBA-140

63 and GYPC; and PfRh4 and CR1), but experimental data suggest their roles in apical

64 reorientation and host cell deformation are largely functionally redundant (Tham et al., 65 2012).

66

67 The only receptor-ligand interaction known to be essential during the pre-invasion phase 68 involves basigin and PfRH5, which exists in a complex with PfRipr and CyRPA (Chen et 
69 al., 2011; Crosnier et al., 2012; Dreyer et al., 2012; Reddy et al., 2015). Binding of the

70 PfRH5 complex to basigin is required for discharge of the rhoptry organelles into the

71 invaded cell and is associated with a calcium spike, potentially due to formation of a pore

72 at the erythrocyte surface (Volz et al., 2016; Weiss et al., 2015). Blocking the interaction

73 between PfRH5 and basigin with specific antibodies prevents invasion (Crosnier et al.,

74 2012).

75

76 Discharge of the rhoptry organelles heralds the start of active invasion. Among the

77 proteins injected from the rhoptries are those of the RON complex (RON 2, RON 4 and

78 RON5), which together form a receptor for binding by the PfAMA1 protein localized on

79 the merozoite surface (Alexander, Arastu-Kapur, Dubremetz, \& Boothroyd, 2006;

80 Alexander, Mital, Ward, Bradley, \& Boothroyd, 2005; Richard et al., 2010). The interaction

81 between PfAMA1 and the RON complex forms a moving junction between the parasite

82 and host cell that is believed to be entirely parasite-derived (Besteiro, Dubremetz, \&

83 Lebrun, 2011; Besteiro, Michelin, Poncet, Dubremetz, \& Lebrun, 2009; Harvey, Yap,

84 Gilson, Cowman, \& Crabb, 2014; Koch \& Baum, 2016). The moving junction provides an

85 anchoring point for the merozoite to actively invade using its own actinomyosin motor;

86 inhibiting the interaction between PfAMA1 and RON prevents invasion (Richard et al.,

87 2010; Srinivasan et al., 2011; Yap et al., 2014).

89 As invasion proceeds, a parasitophorous vacuole is formed from components of the host

90 cell membrane and rhoptries, yielding a protective niche for development of the new

91 daughter parasite. The third phase, echinocytosis, is a transient period of cell dehydration 
92 and shrinkage observed after invasion; current evidence suggests it is triggered by

93 discharge of the rhoptry contents, prior to and independent of active invasion.

94 Echinocytosis is inhibited by reagents that prevent rhoptry discharge, such as antibodies

95 targeting basigin or PfRH5 (Weiss et al., 2015).

97 Most host factors known to play a role in $P$. falciparum invasion have been identified based on their ability to bind to established invasion ligands or from studies of rare natural

99 mutants (Bei \& Duraisingh, 2012). Given the inherent intractability of mature RBCs, which

100 lack a nucleus and DNA, the use of genetic approaches to identify and characterize

101 malaria host factors presents a logistical challenge (Egan, 2017). Recently, an shRNA-

102 based forward genetic screen using cultured red cells (cRBCs) derived ex-vivo from 103 primary human hematopoietic stem/progenitor cells (HSPCs) identified erythrocyte CD55

104 (aka DAF) as a critical host factor for $P$. falciparum invasion (Egan et al., 2015). A 70 kD 105 extracellular glycoprotein anchored to the red cell membrane by a 106 glycosylphosphatidylinositol (GPI) linkage, CD55 is broadly distributed in different tissues 107 and secretions, including blood cells (Cooling, 2015; Storry, Reid, \& Yazer, 2010). On 108 erythrocytes, CD55 acts as a complement regulatory protein to prevent complement109 mediated damage. On epithelial cells, it has been shown to act as a receptor for Group B 110 coxsackie virus and Dr+ E. coli (Cooling, 2015; Coyne \& Bergelson, 2006).

112 We have shown that $P$. falciparum invasion efficiency was reduced by $\sim 50 \%$ in CD55113 knockdown cRBCs, and natural CD55-null erythrocytes from two rare donors with the 114 Inab phenotype were resistant to invasion (Egan et al., 2015). Importantly, the 
115 requirement for CD55 was strain-transcendent, suggesting that it plays a conserved role

116 in P. falciparum invasion. However, the precise function of CD55 during invasion and how

117 it may interface with established ligands or receptors is unknown.

118

119 In this study, we investigated the functional role of CD55 during $P$. falciparum invasion

120 using CRISPR-Cas9 genome editing, antibody-based inhibition and live cell microscopy.

121 We found that CD55 plays a critical role during $P$. falciparum invasion of mature

122 erythrocytes, where it is specifically required for parasite internalization. As a host factor

123 that acts after discharge of the parasite's rhoptry contents, CD55 plays a unique role

124 relative to other receptors required for invasion, providing a crucial link between the

125 ligand-receptor interactions important for adhesion and deformation and effective 126 internalization.

\section{Results}

129 Generation of CD55-null red blood cells using CRISPR-Cas9

130 Previously, we observed that $P$. falciparum merozoites from several laboratory-adapted

131 strains and clinical isolates displayed impaired invasion into cryopreserved CD55-null

132 RBCs from two rare patients with the Inab phenotype (Egan et al., 2015). To study the

133 requirement for CD55 in an isogenic background, we sought to generate CD55-null

134 cRBCs from HSPCs using CRISPR-Cas9 genome editing by co-delivering single guide

135 RNAs (sgRNAs) and Cas9 in a ribonucleoprotein complex (RNP), a method that has been

136 used previously to generate null mutants in primary human CD34+ cells (Hendel et al.,

137 2015). We designed two chemically modified sgRNAs targeting the 5' end of CD55, and 
138 delivered them individually or together into primary human CD34 ${ }^{+}$HSPCs via 139 nucleofection, in complex with recombinant Cas9 (Fig. 1 A). As a control, isogenic CD34

140 HSPCs from the same donor were nucleofected with Cas9 alone. After inducing the cells

141 to differentiate down the erythroid lineage for 18 days, we observed a high percentage of

142 CD55-null cRBCs in the RNP-transfected populations; a single sgRNA targeting exon 1

143 resulted in $\sim 70 \%$ CD55-null cRBCs, while the combination of two sgRNAs targeting exons

1441 and 2 increased the knockout efficiency to $\sim 90 \%$ (Figure 1B). The cells proliferated

14510,000 -fold and the enucleation rate was $>90 \%$ in both the CD55-null cells and wild-type

146 controls, demonstrating that the progenitors differentiated efficiently and that CD55 is not

147 required for this process (Figure S1). The absence of CD55 expression in the mutant 148 cRBCs was also confirmed using immunofluorescence assays (Figure S2). 
A



C

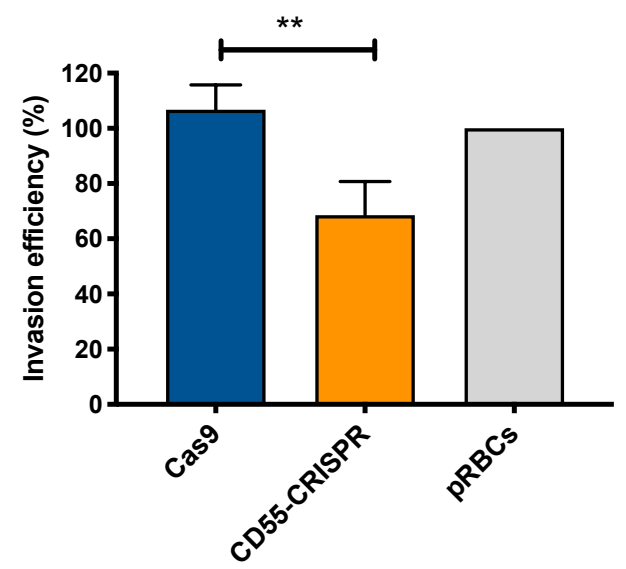

$E$

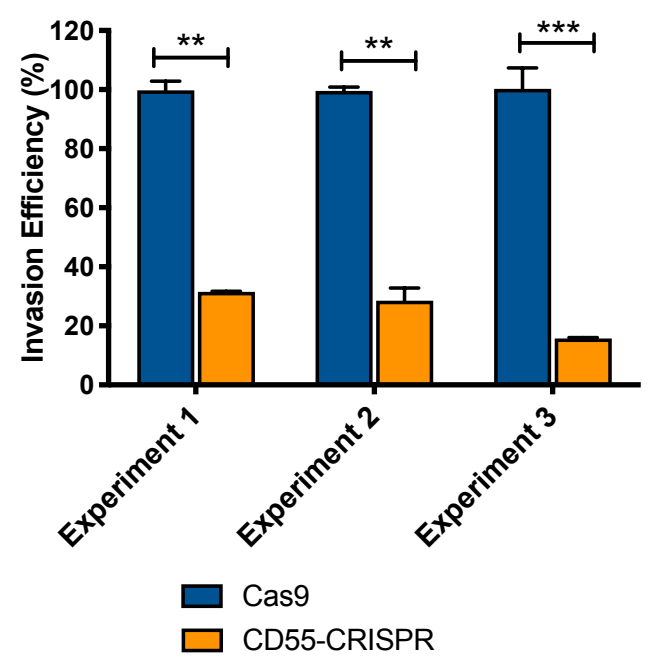

$\mathrm{F}$
B

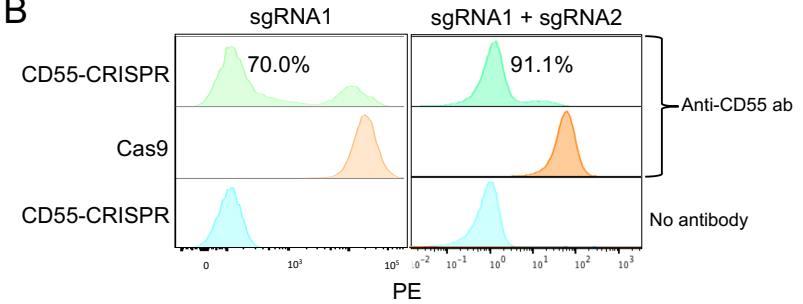

D
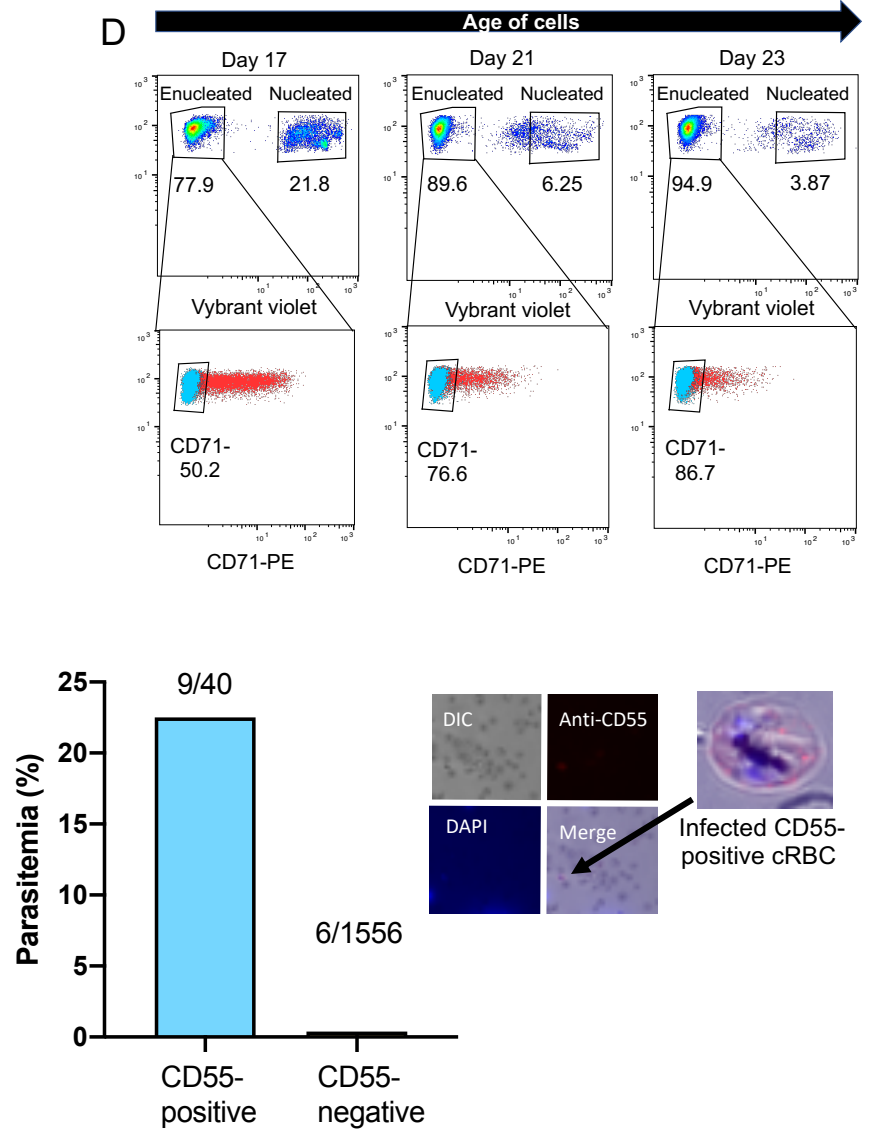

Figure 1: CD55 is required for $\boldsymbol{P}$. falciparum invasion (A) Schematic of CD55 gene structure showing 152 targeting sites of two single guide RNAs (sgRNAs). Vertical red lines indicate the positions of CD55 exons.

153 (B) Expression of CD55 on mutant (CD55-CRISPR) cRBCs generated with one sgRNA (left) or two sgRNAs 154 (right), as compared to control (Cas9) cRBCs. (C) Invasion efficiency of $P$. falciparum 3D7 in Day 18 CD55155 CRISPR cRBCs compared to isogenic controls (Cas9), relative to the invasion efficiency in peripheral blood 156 erythrocytes $\left(\mathrm{N}=4\right.$ biological replicates; $n=3$ technical replicates; error bars indicate $\left.S E M ;{ }^{* *} p<0.01\right)$. (D) 157 Time course of expression of CD71 on cRBCs harvested on different days of differentiation. Enucleated 158 versus nucleated cells were gated using a nuclear dye. (E) Invasion efficiency of $P$. falciparum strain 3D7 159 in CD71-negative, CD55-CRISPR cRBCs compared to CD71-negative isogenic controls (Cas9). Invasion 
160 efficiency is presented relative to the mean of Cas9 control. Three independent biological replicates are 161 shown; error bars represent SEM ( $n=2$ or 3 technical replicates; $\left.{ }^{* *} p<0.01,{ }^{* * *} p<0.005\right)$. (F) Parasitemia in 162 population of CD55-CRISPR cRBCs, in which $~ 90 \%$ of the cells lack CD55 (CD55-negative), and the 163 remaining are CD55-positive, as quantified by immunofluorescence assays. Below are representative 164 images of CD55-CRISPR cRBCs infected with $P$. falciparum. Images are brightfield with fluorescence 165 overlaid. Blue, dapi. Red, anti-CD55-PE. 


\section{CD55 is required for $P$. falciparum invasion}

168 To specifically assess the requirement for CD55 for $P$. falciparum invasion, we performed

169 invasion assays using strain 3D7 in CD55-CRISPR cRBCs or in isogenic control cRBCs

170 that had been differentiated for 18 days. While invasion into the CD55-CRISPR cRBCs

171 was impaired relative to the control cells, it was only reduced by $\sim 40 \%$ (Figure $1 \mathrm{C}$ ). This

172 subtle phenotype was reminiscent of the results for CD55-knockdown cRBCs, and

173 differed from the strong invasion phenotype observed in erythrocytes from two CD55-null

174 patients (Egan et al., 2015). Since cRBCs are less mature than erythrocytes from

175 peripheral blood, we hypothesized that the relatively mild invasion phenotype observed

176 in the CD55-null cRBCs may be explained by the overall maturation state of the ex-vivo

177 cultures on day 18.

178

179 To begin to determine whether cell maturity modifies the requirement for CD55 during $P$.

180 falciparum invasion, we assessed the surface expression of CD71 in the cRBCs, which

181 is highly expressed on erythroblasts and young reticulocytes, but quickly disappears as

182 reticulocytes mature into erythrocytes (J. Hu et al., 2013). Flow cytometry on day 17

183 demonstrated that approximately $50 \%$ of the enucleated cRBCs were CD71-positive and

$184 \sim 50 \%$ were CD71 negative, indicating a mix of cell maturity in the population (Figure 1D).

185 This was further validated using a reticulocyte stain (Figure S3), which showed that day

18617 cells were $\sim 50 \%$ reticulocytes and $50 \%$ erythrocytes. A timecourse over 7 additional

187 days of terminal differentiation revealed a progressive loss of CD71 expression,

188 consistent with a change in the population structure to one dominated by erythrocytes

189 rather than reticulocytes (Figure 1D). 
191 To isolate a more homogeneous population of mature cRBCs for invasion assays, we

192 differentiated the cRBCs for 21-22 days and then used anti-CD71 antibody-immobilized 193 magnetic beads to enrich for CD71-negative cells (Figure S4). Using these highly mature 194 cells, invasion assays with $P$. falciparum strain 3D7 showed a $\sim 75 \%$ reduction in invasion 195 into the CD55-CRISPR cRBCs as compared to isogenic control cRBCs in three biological 196 replicate experiments (Figure 1E). Since our CRISPR-Cas9 genome editing strategy 197 yielded a mixed population of $\sim 90 \%$ CD55-null and 10\% CD55-positive cells, we 198 suspected that the residual invasion observed in the "CD55-CRISPR" population was 199 attributable to wild-type cells. This was confirmed by immunofluorescence assays 200 showing that $P$. falciparum invasion was restricted to the cells expressing CD55: the 201 parasitemia in the minority, CD55-positive cells in the population was $22.5 \%$, whereas 202 less than $0.5 \%$ of the CD55-null cRBCs were infected (Figure 1F). These results 203 demonstrate that CD55 is critical for $P$. falciparum invasion of fully differentiated cRBCs, 204 mimicking the prior observations with natural CD55-null erythrocytes, and validating 205 CD55 as an essential host factor for $P$. falciparum invasion. Based on previous 206 observations that the expression levels of many RBC surface proteins decline during 207 reticulocyte maturation (Chu et al., 2018; Malleret et al., 2013), we hypothesize that high 208 levels of other receptors important for invasion, such as basigin, may explain the reduced 209 requirement for CD55 for $P$. falciparum invasion of younger, CD71-positive reticulocytes. 
212 To determine if $P$. falciparum invasion can be inhibited using antibodies targeting CD55,

213 we performed in vitro growth assays in the presence of three established anti-CD55

214 monoclonal antibodies individually and in combination. The antibodies each recognize a

215 different short consensus repeat (SCR) in the CD55 ectodomain: BRIC 230, BRIC 110

216 and BRIC 216 target SCR1, SCR2 and SCR3, respectively. While none of the antibodies

217 individually affected parasite growth, we observed a dose-dependent inhibition of parasite

218 growth over 72 hours in the presence of all three anti-CD55 monoclonal antibodies

219 combined, in comparison to an isotype control (Figure 2A). At the highest antibody

220 concentration tested $(500 \mu \mathrm{g} / \mathrm{ml})$, the relative parasitemia was reduced by $\sim 40 \%$ in the

221 presence of the combined monoclonals, compared to the isotype control. 

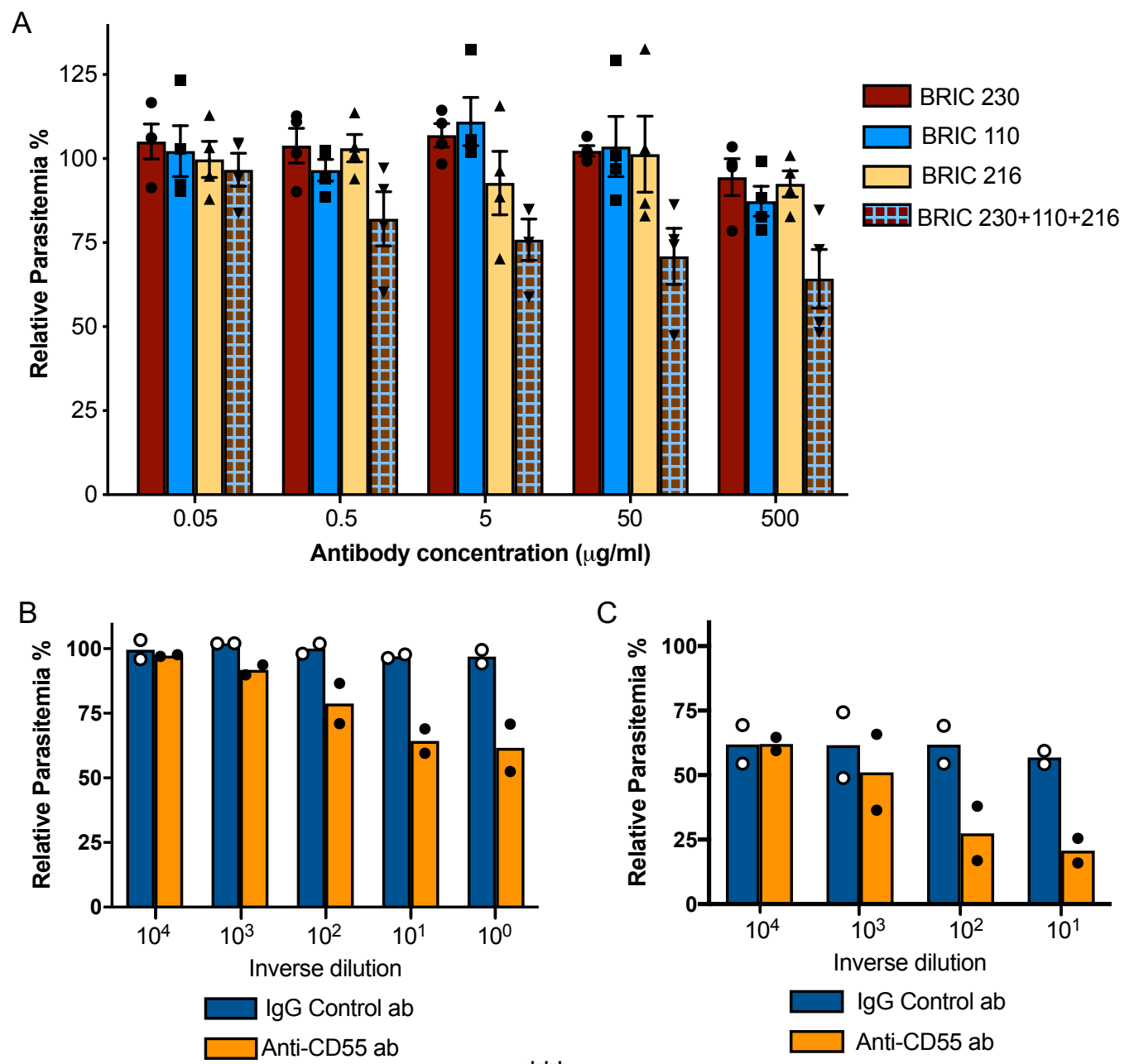

C
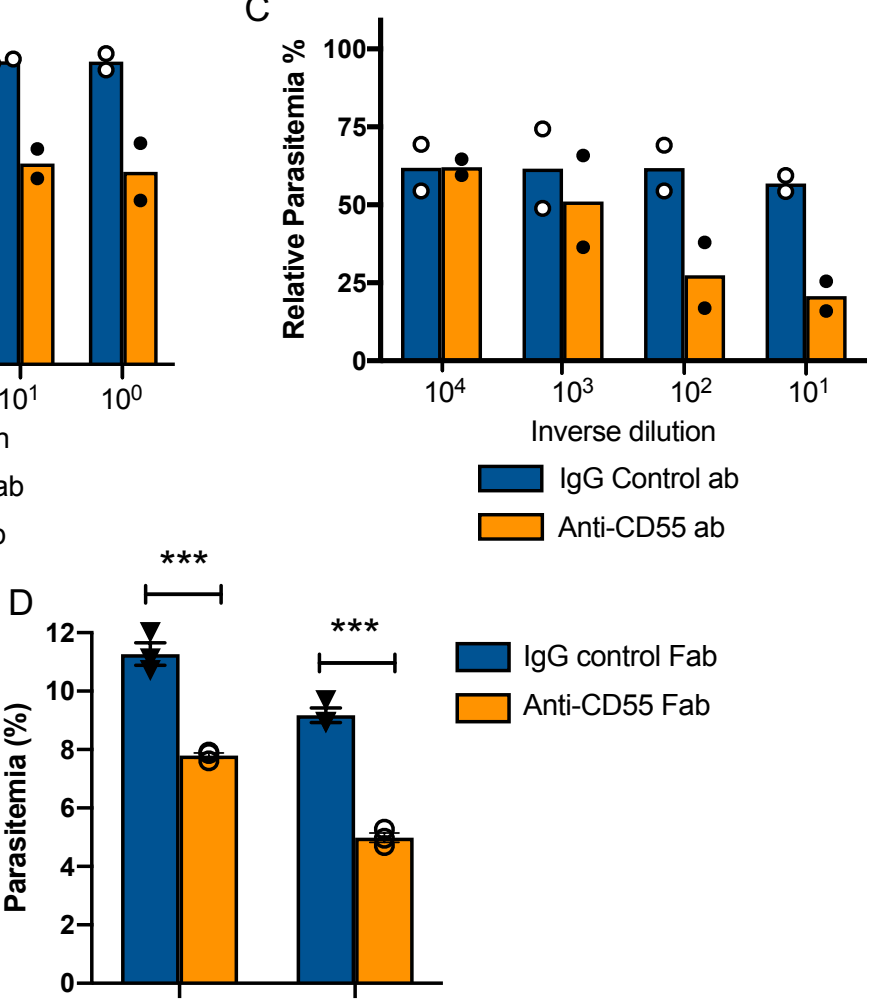

IgG Control ab Anti-CD55 ab

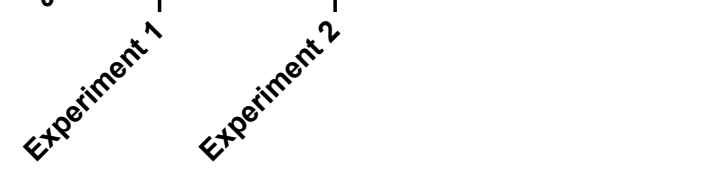

223 Fig 2: Blocking CD55 with antibody inhibits growth of $P$. falciparum (A) $P$. falciparum strain 3D7

224 parasitemia after 72 hours of growth in RBCS in the presence of increasing concentrations of anti-CD55 monoclonal antibodies, relative to isotype control antibody (BRIC 170) at the same concentration. For the pooled antibodies, the indicated concentration was the total combined value, and there were equimolar

227 amounts of each antibody. ( $N=3$ biological replicates; $n=2$ technical replicates). Error bars indicate SEM. 
228 (B) Parasitemia of $P$. falciparum strain 3D7 after 72 hours growth in non-enzyme treated RBCs with 229 increasing concentrations of polyclonal anti-CD55 IgG antibody, relative to that in isotype control antibody 230 at same concentration. ( $N=2$ biological replicates; $n=2$ technical replicates). The highest antibody 231 concentration $\left(10^{\circ}\right)$ was $400 \mathrm{ug} / \mathrm{ml}$. (C) As in B, but with neuraminidase-treated RBCs. The highest antibody 232 concentration $\left(10^{1}\right)$ was $40 \mathrm{ug} / \mathrm{ml}$. (D) $P$. falciparum strain 3D7 parasitemia after 72 hours growth in 400 $233 \mathrm{ug} / \mathrm{ml}$ Fab fragments generated from anti-CD55 polyclonal antibody or isogenic control. Error bars indicate 234 SEM. ${ }^{* * *} p<0.005$. The starting parasitemias were $0.3 \%$ (Experiment 1 ) or $0.5 \%$ (Experiment 2 ).

235 
237 To further study anti-CD55-mediated inhibition of $P$. falciparum invasion, we generated a 238 rabbit polyclonal antibody raised against the entire ectodomain of human erythrocyte

239 CD55. Flow cytometry analysis confirmed that the purified IgG antibody recognizes an 240 antigen on wild-type (WT) RBCs but not on CD55-null RBCs from an Inab donor

241 (Takahashi, 2008), confirming its specificity for CD55 (Figure S5). In P. falciparum growth

242 assays, we observed a dose-dependent inhibition of parasite growth in the presence of 243 anti-CD55 antibody relative to isotype control, with a $\sim 40 \%$ reduction in relative

244 parasitemia at the highest concentration of antibody $(400 \mu \mathrm{g} / \mathrm{ml})$ (Figure $2 \mathrm{~B})$. This degree 245 of inhibition was very similar to that seen for the pooled monoclonal antibodies; together, 246 these findings suggest that blocking CD55 on the RBC can inhibit $P$. falciparum invasion.

248 We next tested the effect of anti-CD55 antibody on sialic acid-independent invasion 249 pathways by treating cells with neuraminidase (NM) to remove sialic acid. As has been 250 described previously, the growth of $P$. falciparum strain 3D7 was inhibited $\sim 40 \%$ in NM251 treated RBCs as compared to untreated cells in the absence of antibody (Figure 2C), 252 reflecting some reliance of strain 3D7 on sialic acid for efficient invasion. In the presence 253 of increasing concentrations of anti-CD55 antibody, we observed a dose-dependent 254 inhibition of parasite growth in the NM-treated cells that is similar to untreated cells ( $60 \%$ 255 versus $\sim 40 \%$ at maximum concentration of antibody) (Figure $2 \mathrm{~B}-\mathrm{C}$ ). The finding that 256 CD55 blockade inhibits $P$. falciparum regardless of the presence of membrane sialic acid 257 suggests that CD55 plays a role in both sialic acid-dependent and -independent invasion. 258 These results are consistent with previous findings showing that the requirement for CD55 
259 is strain-transcendent, including strains that rely on sialic acid to various degrees (Egan

260 et al., 2015).

261

262 To confirm that the inhibitory effect of the polyclonal anti-CD55 antibody on $P$. falciparum

263 growth was not due to crosslinking, we tested the growth inhibitory activity of monovalent

264 anti-CD55 antibody fragments (Fab fragments). In the presence of $400 \mu \mathrm{g} / \mathrm{ml}$ of anti-CD55

265 Fab fragments, we observed $\sim 40 \%$ reduction in relative parasitemia, recapitulating the

266 growth inhibitory activity observed with the bivalent anti-CD55 IgG antibody (Figure 2D).

267 These results further support the conclusion that CD55 is a critical host receptor for $P$.

268 falciparum.

269

270 Blocking CD55 inhibits $P$. falciparum internalization

271 To specifically determine the impact of CD55 blockade on $P$. falciparum invasion, we used

272 live cell imaging to visualize and quantify schizont rupture and merozoite invasion in real

273 time in the presence of the anti-CD55 polyclonal antibody or an isotype control (Figure

$2743 \mathrm{~A}$; Movies S1-S2). First, we quantified the efficiency of merozoite internalization in the

275 presence of anti-CD55 antibody versus isotype control. In the presence of the isotype

276 control antibody, of $345 P$. falciparum merozoites that contacted an RBC, 53 invaded

277 successfully (15\%) (Figure 3B). This frequency is similar to that described in previous live

278 microscopy studies analyzing the efficiency of 3D7 merozoite invasion in the absence of

279 antibodies (Volz et al., 2016; Weiss et al., 2015). In comparison, merozoite invasion was

280 significantly reduced in the presence of anti-CD55 antibody. Out of 312 merozoites that

281 made contact with an RBC in the presence of anti-CD55, only 21 successfully invaded 
$282(6.7 \%)$ (Figure 3B). The number of invasion events per schizont rupture was similarly 283 reduced by half in the presence of anti-CD55 compared to control antibody (Figure 3C),

284 indicating that anti-CD55 antibody inhibits entry but does not prevent initial contact

285 between the merozoite and RBC.

286

287 Blocking CD55 has no impact on pre-invasion kinetics

288 Next, we examined the impact of CD55 blockade on the kinetics of the three main phases

289 of $P$. falciparum invasion: the pre-invasion time (period from initial contact to the onset of 290 internalization), the internalization time, and the time to echinocytosis (transient period of 291 cell dehydration that occurs after internalization) (Dvorak, Miller, Whitehouse, \& Shiroishi, 292 1975; Weiss et al., 2015). For the subset of merozoites that ultimately invaded 293 successfully, there was no difference in the length of the pre-invasion time, internalization

294 time, or time to echinocytosis in the presence of anti-CD55 antibody compared isotype 295 control (Figure 3D-F). These results indicate that blocking CD55 does not impact pre296 invasion kinetics, at least not for the merozoites that manage to invade in the presence 297 of antibody. 
A

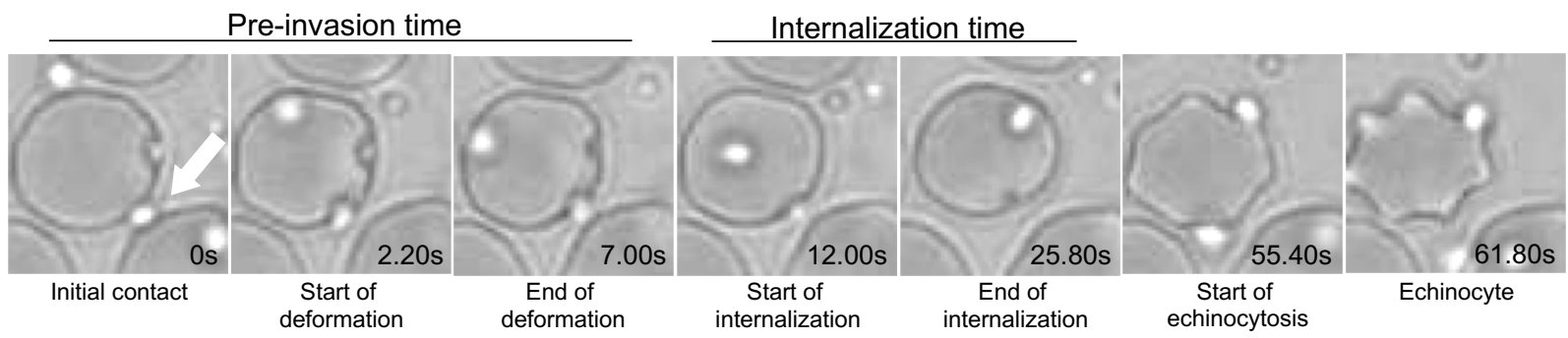

B

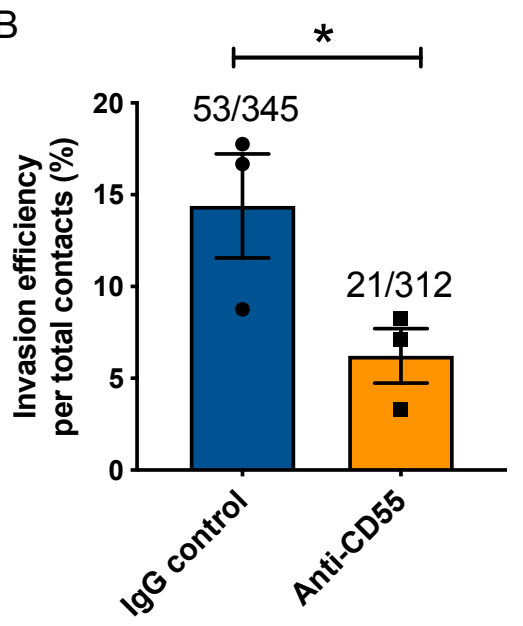

E

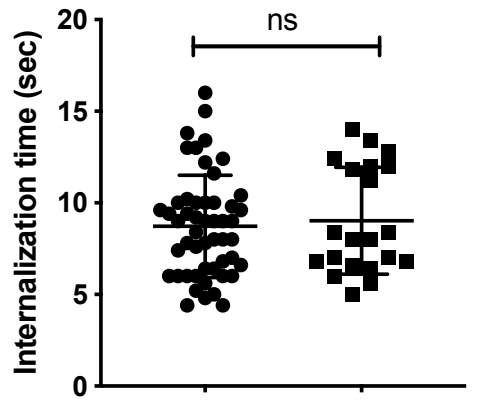

C

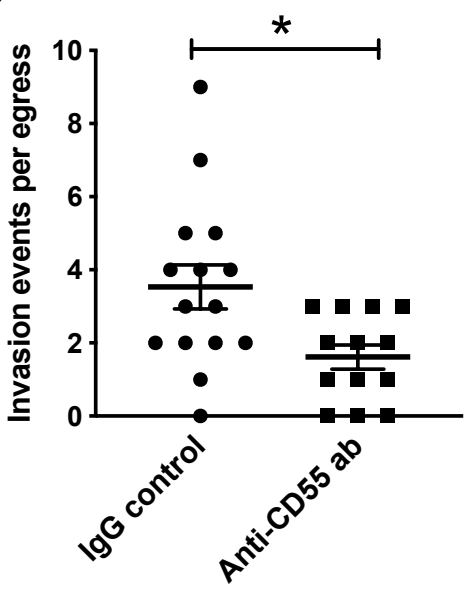

D

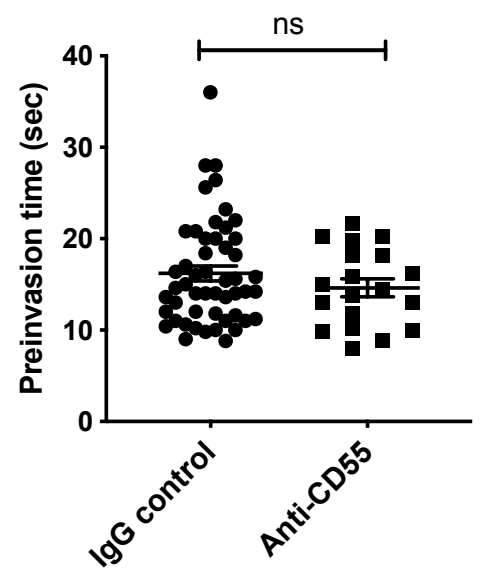

F

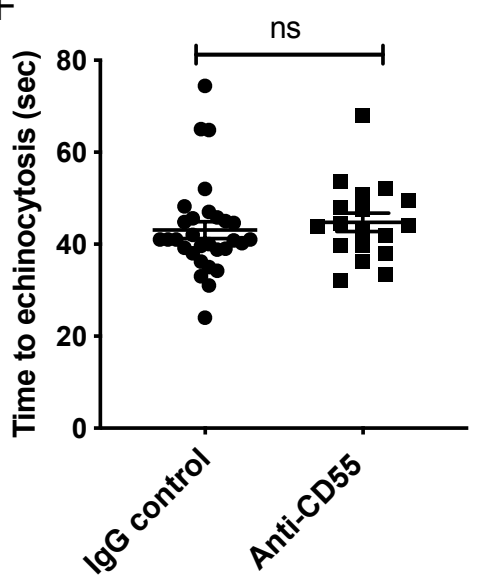

Fig 3: Blocking CD55 with antibody inhibits invasion but not pre-invasion kinetics (A) Time-lapse images of invasion after initial merozoite contact. Arrowhead indicates invading merozoite. Time in seconds. (B) Percentage of merozoites that invaded an RBC after initial contact in presence of polyclonal anti-CD55 antibody (anti-CD55) or isotype control (IgG control). Bottom number is the total number of merozoites followed that made contact with the RBC, and top number is the subset that invaded. The data were acquired in three independent experiments, and the dots indicate the mean invasion efficiency from each experiment; ${ }^{*} p=0.03$. (C) Number of successful invasion events per schizont rupture (egress) in presence of anti-CD55 antibody or isotype control; ${ }^{*} p=0.01$. (D) Pre-invasion time (in seconds) for merozoites that 
bioRxiv preprint doi: https://doi.org/10.1101/2020.07.27.223644; this version posted July 28, 2020. The copyright holder for this preprint (which was not certified by peer review) is the author/funder, who has granted bioRxiv a license to display the preprint in perpetuity. It is made available under aCC-BY-NC-ND 4.0 International license.

308 contact an RBC in the presence of anti-CD55 antibody or isotype control._(E) Internalization time (in 309 seconds) for merozoites in the presence of anti-CD55 antibody or isotype control antibody. (F) Time to 310 echinocytosis from the end of merozoite internalization in the presence of anti-CD55 antibody or isotype 311 control. Error bars indicate SEM; ns, not significant (B-F).

312 


\section{Merozoite-induced erythrocyte deformation is unaffected by CD55 blockade}

314 During the pre-invasion period, parasite attachment to the RBC is associated with

315 substantial deformation of the host cell membrane as the merozoite reorients apically

316 (Dvorak et al., 1975; Gilson \& Crabb, 2009; Paul et al., 2015). The degree of deformation

317 is variable, and is mediated by interactions between parasite ligands released from the

318 apical microneme organelles, such as the EBAs and Rhs, and receptors on the RBC

319 membrane (Weiss et al., 2015). To investigate whether CD55 plays a role in RBC

320 deformation, we used live microscopy to quantitate the efficiency and kinetics of

321 merozoite-induced RBC deformation in the presence of anti-CD55 antibody versus

322 isotype control. The efficiency of deformation was similar in the presence of the two

323 antibodies: approximately $67 \%$ of merozoites deformed the RBC in the presence of anti-

324 CD55 antibody, compared to $\sim 60 \%$ deformation efficiency with the isotype control (Figure

$3254 \mathrm{~A})$. In addition, there was no significant difference in the duration of the deformation

326 period in the presence of the two different antibodies (Figure 4B), suggesting that CD55

327 does not influence merozoite-induced deformation. 
A



B

D
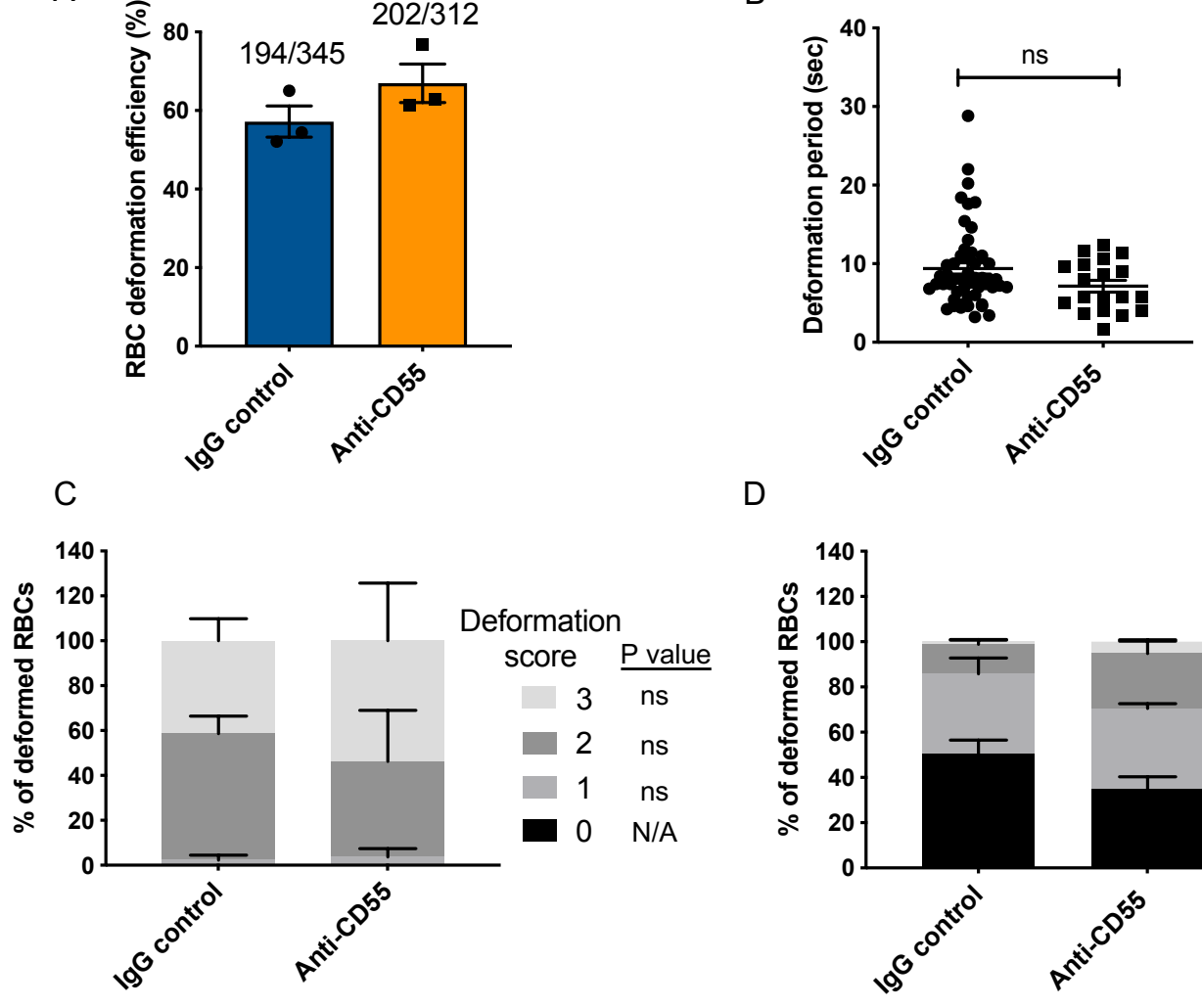

329 Fig 4: Merozoite-induced erythrocyte deformation is not affected by blocking CD55 (A) Efficiency of

330 RBC deformation by merozoites that make contact in presence of anti-CD55 or lgG control. The fractions 331 indicate the number of deformed RBCs out of the total merozoite-RBC contacts observed. The data were 332 acquired in three independent experiments, and the dots represent the mean for each experiment.; * $p=0.02$.

333 (B) Duration of erythrocyte deformation induced by attached merozoites that ultimately invaded in presence 334 of polyclonal anti-CD55 antibody (anti-CD55) or isotype control (IgG control). Time in seconds. (C-D) 335 Strength of merozoite-induced deformation in presence of anti-CD55 or isotype control for cases where 336 invasion was successful (C) or not successful (D); N/A, not applicable; ns, not significant, ${ }^{*} p=0.0029$. Error 337 bars indicate SEM (A-D). 
339 We employed a four-point deformation scale to quantify the intensity of merozoite-induced

340 RBC deformation in the presence of antibody, where 0 denotes the absence of

341 deformation and 3 denotes the most extreme degree of deformation (Weiss et al., 2015)

342 (Movies S3-S5). Almost all merozoites that invaded successfully induced strong

343 deformation (scores of 2 or 3 ), regardless of which antibody was present (Figure 4 C). In

344 comparison, merozoites that failed to invade had lower deformation scores, the majority

345 having scores of 0 or 1 . For these merozoites, the distribution of scores skewed higher

346 in the presence of anti-CD55 antibody compared to the isotype control, perhaps reflecting

347 the inability of otherwise "fit" merozoites to complete invasion (Figure 4D). Taken

348 together, these findings demonstrate that blocking CD55 with antibody does not influence

349 the efficiency, duration, or strength of merozoite-induced RBC deformation, and instead

350 suggest that CD55 exerts its effect on invasion at a downstream step.

352 To further validate the conclusions of the antibody inhibition experiments, we took a 353 complementary approach and performed live cell imaging of $P$. falciparum invasion with 354 natural CD55-null erythrocytes from a rare donor with the Inab phenotype, where CD55 355 is absent due to a stop codon in exon 2(Takahashi, 2008). In traditional invasion assays, 356 we observed $\sim 80 \%$ reduction in the efficiency of $P$. falciparum invasion into CD55-null 357 RBCs as compared to control RBCs after 18 hours, as has been shown previously 358 (Egan et al., 2015) (Figure S6). When imaging invasion in real time, we did not observe 359 any successful invasion events into the CD55-null RBCs, out of 310 merozoites that made 360 contact (Figure 5A and Movies S6-S7). In comparison, there were 25 successful invasion 361 events into the control RBCs, out of 197 merozoites that made contact $(\sim 11 \%)$. Although 
362 invasion into the CD55-null cells was clearly impaired, there was no difference in the

363 efficiency of merozoite-induced RBC deformation between the two genetic backgrounds

364 (Figure 5B). Moreover, we observed no significant difference in the distribution of

365 deformation scores between CD55 null and control RBCs for merozoites that did not

366 invade (Figure 5C). These results are consistent with the findings from the antibody

367 inhibition experiments, and suggest that CD55 influences invasion by acting after the 368 period of erythrocyte deformation.

A

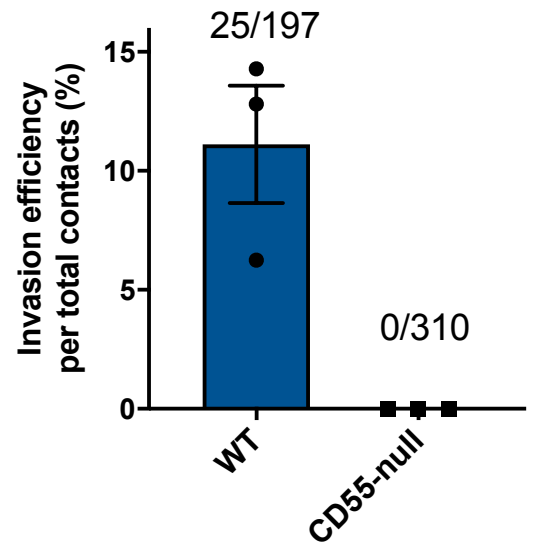

B

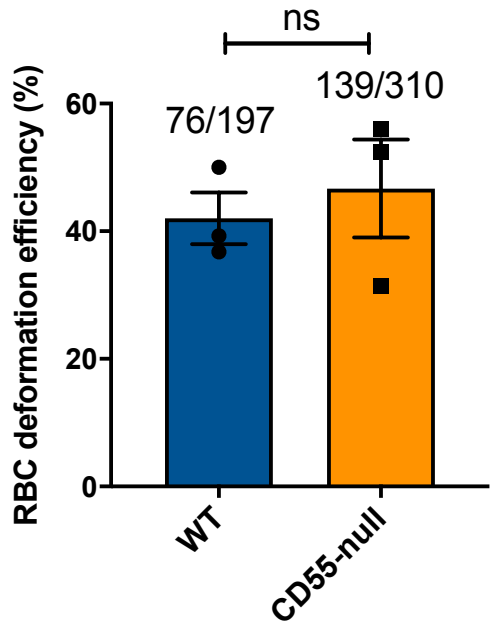

C

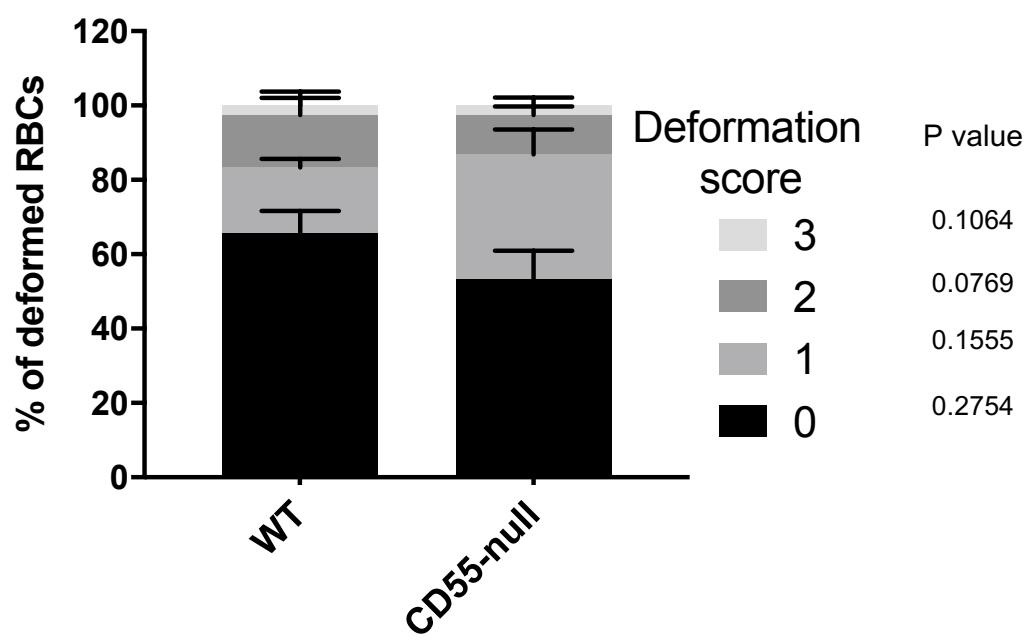

Fig 5: Absence of CD55 prevents invasion but does not impact deformation. (A) Percentage of 
372 number of merozoites followed that made contact with the RBC, and top number is the subset that invaded.

373 The data were acquired in three independent experiments, and the dots indicate the mean invasion

374 efficiency from each experiment; ${ }^{*} p=0.04$. (B) Efficiency of WT or CD55-null RBC deformation upon

375 merozoite contact. Bottom number is the total number of RBCs contacted by a merozoite, and top number

376 is the subset that were deformed upon contact. The data were acquired in three independent experiments,

377 and the dots indicate the mean deformation efficiency for each experiment; ns, not significant. (C) Strength

378 of merozoite-induced deformation of WT or CD55-null RBCs among non-invading merozoites; ns, not 379 significant. Error bars indicate SEM (A-C). 


\section{CD55 acts downstream of rhoptry discharge}

383 Since we observed that the blocking or deletion of CD55 impaired $P$. falciparum entry without altering pre-invasion kinetics or membrane deformation, we hypothesized that

385 CD55 functions after the ligand-receptor interactions that mediate attachment and deformation. During the sequential steps of $P$. falciparum invasion, RBC deformation is

387 followed by injection of the rhoptry organelle contents into the host cell cytoplasm, an event that requires an interaction between the parasite ligand PfRh5 and its RBC receptor, basigin (Weiss et al., 2015). The contents of the rhoptries in turn are believed to trigger echinocytosis. When the interaction between PfRH5 and basigin is blocked using inhibitory antibodies and rhoptry discharge is prevented, echinocytosis fails to occur

392 (Volz et al., 2016; Weiss et al., 2015). To investigate whether CD55 is similarly required for release of the rhoptry contents, we quantified the incidence of RBC echinocytosis

394 elicited by attached merozoites in the presence of anti-CD55 antibody versus isotype 395 control using a cytochalasin-D (cyt-D) live microscopy assay (Figure 6A-B and Movie S8). Cyt-D is an inhibitor of actin polymerization that prevents merozoite internalization by 397 inhibiting the actino-myosin motor, but does not impact attachment, rhoptry discharge, or 398 echinocytosis (Miller, Aikawa, Johnson, \& Shiroishi, 1979; Weiss et al., 2015). Upon 399 treatment with cyt-D, $\sim 60 \%$ of merozoites that attached to an RBC triggered echinocytosis 400 in the presence of the isotype control antibody, and a similar rate of echinocytosis was 401 observed in the presence of anti-CD55 antibody (Figure 6C). These results demonstrate 402 that blocking CD55 does not significantly impact rhoptry discharge or echinocytosis, 403 standing in distinct contrast to what has been observed for basigin and Rh5, where 404 blocking their interaction with antibodies prevents echinocytosis (Weiss et al., 2015). 
bioRxiv preprint doi: https://doi.org/10.1101/2020.07.27.223644; this version posted July 28, 2020. The copyright holder for this preprint (which was not certified by peer review) is the author/funder, who has granted bioRxiv a license to display the preprint in perpetuity. It is made available under aCC-BY-NC-ND 4.0 International license.

405 Together, these findings demonstrate that CD55 acts downstream of the interaction

406 between $\mathrm{RH} 5$ and basigin, facilitating a step of invasion that occurs after release of the

407 rhoptry organelles. 


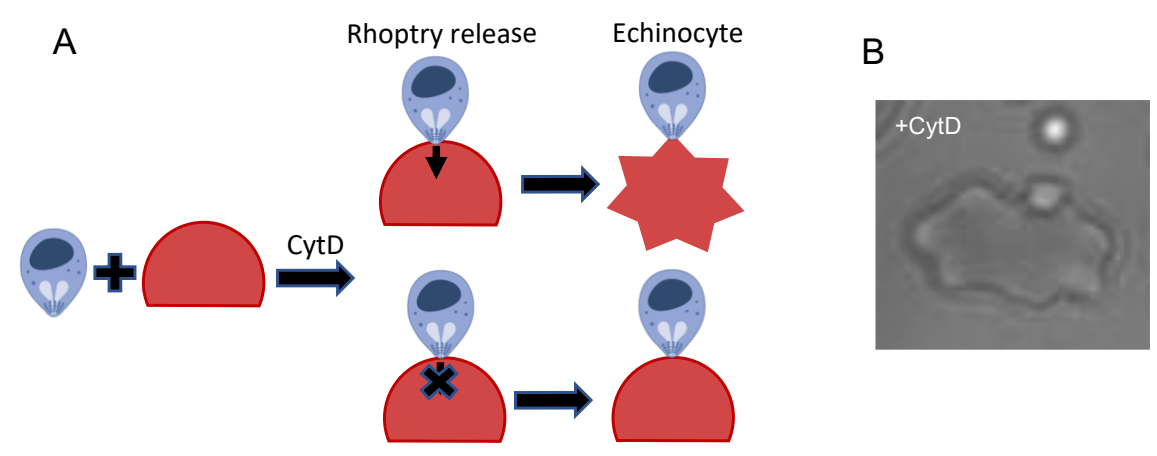

C

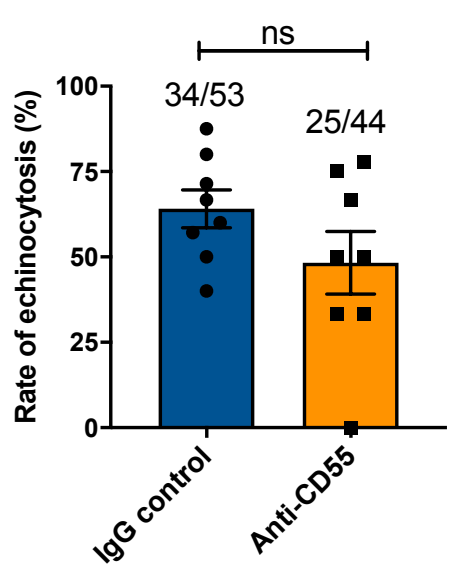

Fig 6: Blocking CD55 with antibody does not impact echinocytosis. (A) Cartoon illustrating echinocytosis elicited by an attached merozoite that has discharged its rhoptry contents in the presence of cyt-D, which prevents internalization. Reagents that block rhoptry discharge prevent echinocytosis. (B) Image showing an echinocyte with an attached merozoite. Scale bar=5um. (C) Echinocytosis efficiency in the presence of antiCD55 antibody or isotype control. Bottom number is the total number of merozoite-RBC pairs observed to make contact, and top number is the subset in which echinocytosis occurred. The data are from eight independent experiments. Dots indicate the mean from each experiment and error bars represent the standard error of mean; ns, not significant. 


\section{CD55 may be required for progression of the moving junction}

411 The moving junction that forms between the cell membranes of an invading merozoite

412 and the erythrocyte during internalization involves interactions between PfAMA1, which

413 derives from the micronemes, and the PfRON complex, which is expressed by the

414 rhoptries. To investigate a possible role for CD55 in formation of the moving junction, we

415 sought to visualize PfAMA1 and PfRON4 as merozoites attempted to invade CD55-null

416 (Inab) or wild-type erythrocytes in the presence of cyt-D. Under these conditions, we

417 observed a $\sim 50 \%$ reduction in the efficiency of merozoite attachment to CD55-null

418 erythrocytes as compared to WT controls by flow cytometry, as has been observed

419 previously (Figure 7A) (Egan et al., 2015). Using confocal microscopy, we found that

420 PfAMA1 and PfRON4 were frequently co-localized at the cellular interface for merozoites

421 attached to WT control cells ( 85\%) (Figure 7B). In comparison, for merozoites attached

422 to CD55-null erythrocytes, PfAMA1 and PfRON4 were co-localized at the cellular

423 interface in only $\sim 40 \%$ of cases (Figure $7 B$ ). For those merozoites in which PfAMA1 and

424 PfRON4 were colocalized at the interface, we observed that approximately half of the WT

425 control cells had an indentation at the point of merozoite contact, suggesting the

426 merozoites were mid-invasion, whereas this was never observed for the CD55-null cells

427 (Figure 7C). Together, these results support a model where CD55 is required for 428 progression of the moving junction, and acts downstream of and distinctly from all other

429 known host receptors for $P$. falciparum invasion. 
A



C
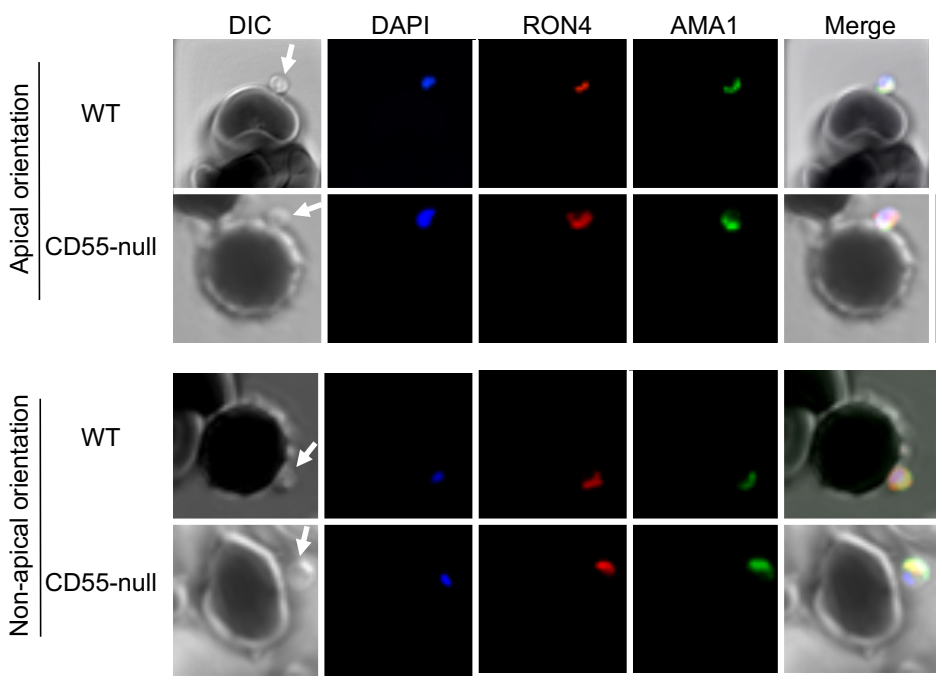

D
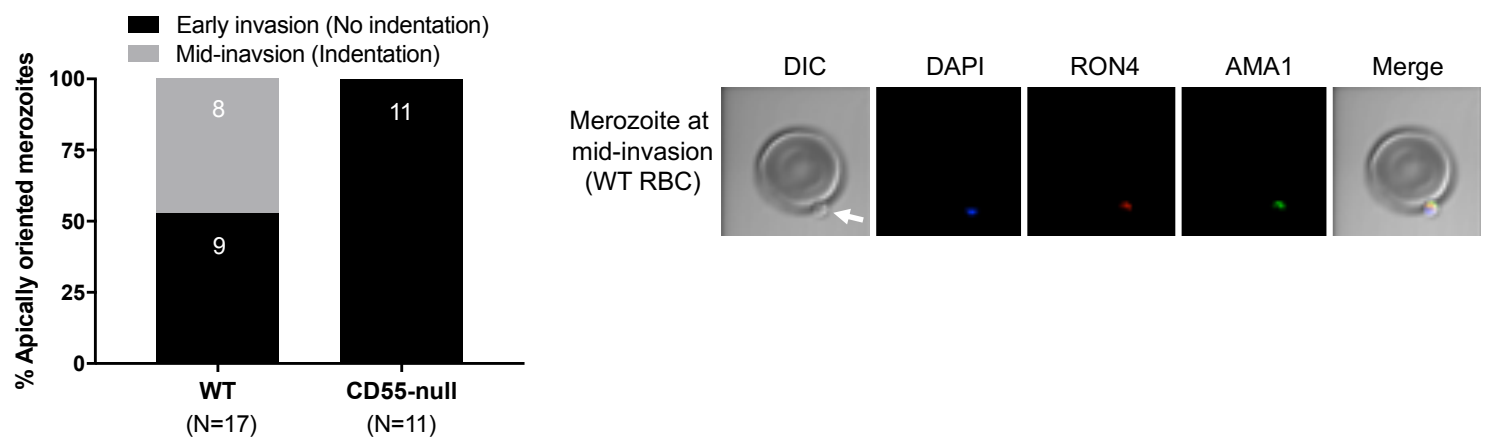

431 Fig 7: Invasion fails to progress in the absence of CD55.

432 (A) Attachment efficiency of $P$. falciparum merozoites to CD55-null versus control RBCs in the presence of 433 cyt-D, as measured by flow cytometry 90 min after the addition of synchronized schizonts $(\mathrm{N}=3, \mathrm{n}=3)$. Error 434 bars indicate SEM; * $p<0.018$. (B) Orientation of merozoites attached to WT versus CD55-null RBCs, 435 indicated by the localization of AMA1, RON4, and a density at the merozoite's apical end as measured by 436 confocal microscopy. Apical, AMA1 and RON4 co-localized at the cellular interface and merozoite density 437 abutting RBC; Not apical, AMA1 and RON4 not co-localized at the cellular interface and merozoite density 
bioRxiv preprint doi: https://doi.org/10.1101/2020.07.27.223644; this version posted July 28, 2020. The copyright holder for this preprint (which

was not certified by peer review) is the author/funder, who has granted bioRxiv a license to display the preprint in perpetuity. It is made available under aCC-BY-NC-ND 4.0 International license.

438 not abutting RBC; N.D., indeterminate. The numbers above each bar indicate the subset of attached 439 merozoites in that orientation. (C) Representative confocal images showing merozoites attached to RBCs 440 with apical or non-apical orientation. Merozoite density at the apical end of the merozoite is indicated by an 441 arrow. (D) Progression of invasion for merozoites apically attached to CD55-null or WT RBCs in the 442 presence of cyt-D. Panel on the right shows representative confocal images of a merozoite at the mid443 invasion stage. 


\section{Discussion}

446 A comprehensive understanding of the molecular interactions required for $P$. falciparum

447 invasion has been limited by the absence of a robust genetic system to study red cells,

448 which are terminally differentiated and lack a nucleus and DNA. Previously, an RNAi-

449 based forward genetic screen in cultured red cells derived from HSPCs identified two new

450 candidate host factors for invasion: CD44 and CD55 (Egan et al., 2015). In this study, we

451 used a combination of genetics and inhibitory antibodies to determine the precise steps

452 of $P$. falciparum invasion during which CD55 functions. We have demonstrated that CD55

453 is specifically required for merozoite internalization, and plays a unique role relative to the

454 other host receptors known to act during invasion.

$455 \quad$ Prior work has shown that the efficiency of $P$. falciparum infection is reduced by $\sim$

$45650 \%$ in CD55-deficient cRBCs where expression is downregulated using shRNAs. While

457 experiments using erythrocytes from two rare, CD55-null donors suggested that CD55 is

458 essential for $P$. falciparum invasion, this has never been demonstrated genetically. A

459 major roadblock to such experiments has been the technical challenges associated with

460 CRISPR-Cas9 genome editing in primary human hematopoietic stem cells, including

461 cytotoxicity from nucleic acids and low rates of transfection (Hendel et al., 2015). In this

462 study, we showed for the first time that fully mature, CD55-null cRBCs can be generated

463 efficiently from primary human HSPCs using CRISPR-Cas9 genome editing. Using

464 isogenic wild-type and mutant cells, we have demonstrated that CD55-null cRBCs are 465 resistant to $P$. falciparum invasion, confirming that CD55 is an essential host factor for $P$. 466 falciparum. 
468 Here, we demonstrated the feasibility and benefits of generating truly mature erythrocytes 469 ex-vivo for the study of malaria host factors, as these cells closely mimic the target cell

470 for $P$. falciparum in the human bloodstream. Our approach involving the co-delivery of

471 two chemically modified sgRNAs together with Cas9 as a ribonucleoprotein complex has

472 been shown to be an effective strategy for CRISPR-based gene knockout in primary

473 HSPCs and T cells (Hendel et al., 2015). This method obviates the toxicity associated 474 with plasmid delivery, minimizes off-target activity, and improves sgRNA stability.

475 Combined with our ex-vivo erythropoiesis culture system, this method can efficiently 476 generate terminally differentiated, enucleated, CD71-low red cells with a high rate of gene

477 knock out that can be used to study host genetic determinants for $P$. falciparum in an 478 isogenic background.

480 We observed that the reliance of $P$. falciparum on CD55 for invasion increased 481 significantly as enucleated cRBCs matured into erythrocytes, likely reflecting the 482 substantial changes in protein abundance that occur during terminal maturation of human 483 red cells, including for proteins known to act as receptors, such as basigin and CR1 484 (Gautier et al., 2016; G. Hu et al.). The efficiency of $P$. falciparum invasion is further 485 influenced by the deformability of the red cell membrane (Tiffert et al., 2005), a biophysical 486 property that also changes as red cell progenitors proceed through enucleation and final 487 maturation (Giarratana et al., 2005). As clinical malaria is primarily a disease of mature, 488 peripheral blood erythrocytes, our results showing that terminal red cell maturation 489 modifies the requirement for CD55 during invasion highlights the potential drawbacks of 490 using cell lines to study host factors for $P$. falciparum. While considered erythroid in 
491 nature, immortalized cell lines such as JK-1, EJ and BEL-A have low rates of enucleation

492 and terminal maturation (Kanjee et al., 2017; Satchwell et al., 2019; Scully et al., 2019),

493 suggesting their utility for genetic experiments on $P$. falciparum invasion may be limited

494 by incomplete phenotypes.

496 Antibodies targeting a variety of specific host receptors for $P$. falciparum have been 497 shown to have invasion inhibitory activity, including those against GYPA, CR1, and 498 basigin (Crosnier et al., 2012; Pasvol et al., 1989; Spadafora et al., 2010). While we found 499 that individual monoclonal antibodies against three distinct SCR domains of CD55 had no discernable effect on $P$. falciparum growth, in combination they had potent dose501 dependent inhibitory activity, suggesting CD55's role in invasion is not restricted to a 502 specific SCR domain. Our demonstration that both the polyclonal anti-CD55 antibody and 503 anti-CD55 Fab fragments can inhibit $P$. falciparum growth aligns with the results from our 504 genetic studies, and corroborates the conclusion that CD55 is a critical host factor for $P$. 505 falciparum. In contrast to CR1, where antibodies are only inhibitory in the absence of red 506 cell sialic acid (Spadafora et al., 2010), anti-CD55 antibody blocked both sialic acid507 dependent and -independent invasion. This finding is consistent with prior studies 508 showing that the requirement for CD55 in $P$. falciparum invasion is strain-transcendent 509 (Egan et al., 2015), and implies a model where CD55 acts distinctly from CR1 and the 510 other alternative, strain-specific receptors.

512 Given the inherent limitations associated with studying invasion on a population scale in 513 longer term assays, live microscopy has been increasingly employed to visualize 
514 individual $P$. falciparum invasion events in real time. Analysis of the morphology and

515 kinetics of discrete invasion steps in the context of blocking antibodies or soluble proteins

516 has contributed to a model describing the molecular events that occur during invasion

517 (Volz et al., 2016; Weiss et al., 2015). Our live microscopy experiments have added a

518 new dimension to this model by revealing that merozoite internalization is inhibited in the

519 presence of anti-CD55 antibody or with CD55-null erythrocytes, demonstrating for the first

520 time that CD55 is specifically required for parasite entry. Where and how does CD55 act

521 in relation to the $P$. falciparum ligands and erythrocyte receptors known to function during

522 invasion? We observed no impact of the anti-CD55 antibody on pre-invasion kinetics or

523 merozoite-induced erythrocyte deformability, findings that were validated using CD55-null

524 erythrocytes from a rare donor. These results suggest that CD55 functions distinctly from

525 the "alternative" receptors required for apical reorientation and red cell deformation (e.g.

526 glycophorins and CR1), as blocking interactions between these receptors and their

527 ligands strongly inhibits merozoite-induced deformability (Weiss et al., 2015).

529 Echinocytosis is a phenomenon of transient red cell shrinkage that commences soon after

530 merozoite internalization. Current evidence suggests that it is stimulated by changes in

531 the red cell cytoplasm that occur once an irreversibly-attached merozoite discharges its

532 rhoptry contents into the erythrocyte (Volz et al., 2016; Weiss et al., 2015). Echinocytosis

533 is inhibited by antibodies that block the interaction between PfRH5 and basigin, implying

534 that this interaction is necessary for rhoptry discharge (Weiss et al., 2015). These blocking

535 antibodies also inhibit $\mathrm{Ca}^{2+}$ flux into the erythrocyte, possibly reflecting formation of a pore

536 at the cellular interface when PfRH5 and basigin interact (Volz et al., 2016). The 
537 glycophorins and CR1 act early in the pre-invasion phase during apical reorientation, and

538 blocking their interactions with ligands using genetics, enzyme treatments, or antibody

539 blockade also inhibits echinocytosis (Volz et al., 2016; Weiss et al., 2015). In contrast,

540 echinocytosis is not prevented by treatment with the actin polymerization inhibitor cyt-D

541 or reagents that block the interaction of PfAMA1 and the RON complex. Together, these

542 findings demonstrate that the stimulus for echinocytosis occurs after the interaction of

543 PfRH5 with basigin, but before establishment of the moving junction.

545 Our observation that anti-CD55 antibody had no effect the efficiency of merozoite-induced 546 echinocytosis demonstrates that CD55 acts distinctly from basigin and the other

547 established host receptors known to act during $P$. falciparum invasion. While both anti-

548 CD55 and anti-basigin inhibit invasion, echinocytosis occurs normally in the presence of

549 anti-CD55 but is blocked by anti-basigin antibody, likely due to inhibition of rhoptry 550 discharge (Volz et al., 2016). As blocking CD55 does not inhibit deformation nor

551 echinocytosis during invasion, our findings support a model in which CD55 acts after

552 rhoptry discharge, and specifically impacts merozoite internalization.

554 Merozoite internalization requires the formation of a moving junction between the cell 555 membranes of the invading merozoite and the erythrocyte that moves posteriorly down 556 the parasite as it invades. The current model of the moving junction involves interactions 557 between PfAMA1 expressed on the merozoite surface and the PfRON complex inserted 558 into the red cell membrane, independent of any host-encoded receptors (Alexander et al., 559 2006; Alexander et al., 2005; Besteiro et al., 2009; Richard et al., 2010; Riglar et al., 2011; 
560 Srinivasan et al., 2011). In addition to its key role in internalization, the moving junction

561 has also been proposed to be important for formation of the parasitophorous vacuole (PV)

562 (Yap et al., 2014).

563

564 Could CD55 function as a component of the moving junction during invasion? We

565 observed that PfAMA1 and PfRON4 were almost always co-localized at the cellular 566 interface for merozoites attempting to invade WT cells, whereas this was significantly less

567 common for merozoites attempting to invade CD55-null erythrocytes. For the minority of

568 merozoites that attached to CD55-null erythrocytes and had PfAMA1 co-localized with

569 RON at the cellular interface, none were observed to have progressed past early invasion,

570 unlike those attached to WT cells. Together, these findings support a model in which

571 CD55 is required for progression of the moving junction, either directly or indirectly. Future

572 studies involving higher resolution microscopy such as cryo-EM will be necessary to 573 better characterize how absence of CD55 may impact the moving junction or architecture

574 of the PV after invasion.

575

576 Our findings showing that the function of CD55 during invasion can be narrowed to the

577 internalization step are consistent with a model where CD55 acts distinctly from the other

578 known receptors for invasion, potentially by interacting with a specific parasite ligand. As

579 there is a precedent for CD55 on epithelial cells to act as a pathogen receptor that

580 transmits signals to the host cell (Coyne \& Bergelson, 2006), it is tantalizing to

581 hypothesize that this molecule functions similarly in $P$. falciparum invasion of

582 erythrocytes. Ultimately, biochemical identification of parasite and erythrocyte interaction 
583 partners of CD55 will yield important additional insights into the molecular function of

584 CD55 during merozoite internalization. Given the complexity of $P$. falciparum invasion and

585 the unique and essential role of CD55 relative to other established receptors, targeting its

586 activity or interaction partners in novel intervention strategies may enhance the

587 effectiveness of future therapies or vaccines for malaria.

\section{Methods}

\section{P. falciparum culture, invasion assays and growth inhibition assays}

591 P. falciparum strain 3D7, a laboratory-adapted strain obtained from the Walter and Eliza

592 Hall Institute (Melbourne, Australia) was routinely grown in human erythrocytes (Stanford

593 Blood Center) at 2\% hematocrit in complete RPMI-1640 supplemented with $25 \mathrm{mM}$ 594 HEPES, $50 \mathrm{mg} / \mathrm{L}$ hypoxanthine, $2.42 \mathrm{mM}$ sodium bicarbonate and 0.5\% Albumax 595 (Invitrogen) at $37^{\circ} \mathrm{C}$ in $5 \% \mathrm{CO}_{2}$ and $1 \% \mathrm{O}_{2}$.

597 Parasite invasion assays were performed using synchronized late-stage schizont 598 parasites isolated using a MACS magnet (Miltenyi) and added at $1.0-1.5 \%$ initial 599 parasitemia to the cultured red blood cells (cRBCs) or peripheral red blood cells (pRBCs) 600 at $0.3 \%$ hematocrit. For some invasion assays, cRBCs were enriched for the CD71 601 negative population by using anti-CD71 antibody immobilized magnetic beads (Miltenyi), 602 as described below. Assays were performed in a volume of $100 \mu$ per well in a 96 well 603 plate, or at $50 \mu \mathrm{l}$ per well in Half Area 96-well plates (Corning) for the assays with CD71604 negative cRBCs. The ring stage parasitemia was determined after 18-24 hours by bright605 field microscopy of cytospin preparations stained with May-Grünwald and Giemsa. A 
606 minimum of 1000 cells were counted for each technical replicate. For assays in which

607 there was no selection for CD71-negative cells, the invasion efficiency was determined

608 by normalizing the average ring stage parasitema in each genetic background to the 609 average ring stage parasitemia in control peripheral blood RBCs (pRBCs) for each 610 biological replicate. For assays that used CD71-negative cRBCs, the ring stage 611 parasitema for each genetic background was normalized to the mean for the control 612 cRBCs. Assays were performed at least 3 times using 2-3 technical replicates.

614 Antibody inhibition assays were performed using pRBCs in the presence of mouse 615 monoclonal antibodies obtained from IBGRL (BRIC 216, BRIC 230, BRIC 110, BRIC 616 170), polyclonal anti-CD55 antibody produced by New England Peptide, or isotype control 617 rabbit IgG antibody (Novus). All antibodies were dialyzed overnight in RPMI buffer prior 618 to use. Schizont stage parasites were added to untreated or neuraminidase treated 619 pRBCs at $0.5 \%$ hematocrit at an initial parasitemia of $0.5 \%$ in the presence of 0.05 to 500 $620 \mu \mathrm{g} / \mathrm{ml}$ of the antibodies. For the neuraminidase treatment, the cells were incubated with $62166.7 \mathrm{mU} / \mathrm{ml}$ of neuraminidase from Vibrio cholerae (Sigma) at $37^{\circ} \mathrm{C}$ for $1 \mathrm{~h}$ with shaking 622 and washed 3 times in buffer before use in the growth inhibition assays. Some assays 623 were performed in presence of Fab fragments of anti-CD55 or isotype control IgG 624 antibodies at the concentration of $400 \mu \mathrm{g} / \mathrm{ml}$. The Fab fragments were prepared as 625 described below. Assays were performed 2-3 times with 2-3 technical replicates in a 626 volume of $100 \mu$ per well in 96 well plates. Parasitemias were determined on day 3 by 627 staining with SYBR Green 1 nucleic acid stain (Invitrogen) at 1:2000 dilution in PBS/0.3\% 
628 BSA for 20 minutes, followed by flow cytometry analysis on a MACSQuant flow cytometer

629 (Miltenyi).

\section{Generation of Fab fragments}

632 Polyclonal anti-CD55 antibody and control IgG were digested using the Pierce ${ }^{\mathrm{TM}}$ Fab 633 preparation kit (Thermo Fisher Scientific). The resulting Fab fragments were quantified 634 on a spectrophotometer, concentrated, and buffer exchanged with incomplete RPMI 635 using 30K Amicon Ultra $0.5 \mathrm{ml}$ centrifugal filter (Millipore). For final quantification, the Fab 636 fragments were stained with Coomassie in a 10\% SDS-PAGE gel along with known 637 concentrations of the undigested antibodies, and concentrations of the Fab fragments 638 were determined using ImageJ 1.50i(Schneider, Rasband, \& Eliceiri, 2012).

\section{Generation of cultured red blood cells from primary human CD34+ HSPCs}

641 Bone marrow-derived primary human CD34+ HSPCs (Stem Cell Technologies) were

642 cultured in erythroid differentiation medium (cPIMDM) composed of Iscove Basal Medium 643 (IMDM) (Biochrom) supplemented with 4mM L-Glutamine (Sigma), $330 \mu \mathrm{g} / \mathrm{ml}$ holo644 transferrin (BBI Solutions), $10 \mu \mathrm{g} / \mathrm{ml}$ of recombinant human insulin (Sigma), $2 \mathrm{IU} / \mathrm{ml}$ 645 heparin (Affymetrix), 10-6 M hydrocortisone (Sigma), 100ng/ml SCF (R \& D Systems), 5 $646 \mathrm{ng} / \mathrm{ml} \mathrm{IL-3} \mathrm{(R} \mathrm{\&} \mathrm{D} \mathrm{Systems),} 3 \mathrm{lU} / \mathrm{ml}$ Epo (Amgen) and 5\% plasma (Octapharma) at $37^{\circ} \mathrm{C}$ 647 in 5\% CO2 in air, as previously described (Egan et al., 2015; Giarratana et al., 2011). On 648 the $2^{\text {nd }}$ day of the culture, the cells were subjected to nucleofection with ribonucleoprotein 649 (RNP) complexes as described below. On day 5, the cells were washed and resuspended 650 in fresh cPIMDM to maintain concentration $\sim 1 \times 10^{4} / \mathrm{ml}$. Between day $8-13$, the cells were 
651 maintained in fresh cPIMDM devoid of IL3 and hydrocortisone at concentration of $<5 \times 10^{5}$

652 cells $/ \mathrm{ml}$. On day 13 , the cells were washed and plated at $7.5 \times 10^{5}-1.0 \times 10^{6} \mathrm{cells} / \mathrm{ml}$ in

653 cPIMDM without IL-3, hydrocortisone, or SCF. On day 15-16, the cells were co-cultured

654 at $1.0 \times 10^{6} \mathrm{cells} / \mathrm{ml}$ concentration on a murine stromal cell layer (MS-5), as previously

655 described (Giarratana et al., 2005). The cells were harvested on days 18-22 for different

656 experiments. Growth and differentiation were monitored using hemocytometer-based

657 quantification and light microscopy of cytospin preparations stained with May-Grünwald

658 and Giemsa. To quantify the enucleation rate, cRBCs were incubated in Vybrant

659 DyeCycle violet (Life Technologies) $(1: 10,000)$ at $37^{\circ} \mathrm{C}$ for $30 \mathrm{~min}$, followed by flow 660 cytometry analysis on a MACSQuant flow cytometer (Miltenyi).

661

662 CRISPR-Cas9 genetic modification of primary human CD34+ cells

663 Two sgRNAs targeting human CD55 exons were designed using the Broad Institute's 664 GPP sgRNA design portal and synthesized as chemically modified sgRNAs by Synthego.

665 The sgRNA sequences were: GGGCCCCUACUCACCCCACA, which is predicted to 666 recognize a sequence in exon 1 of $C D 55$, and CUGGGCAUUAGGUACAUCUG, which is 667 predicted to recognize a sequence in exon 2. Ribonucleoprotein (RNP) complexes 668 containing one or both sgRNAs were prepared by slowly adding 300 pmol of each sgRNA 669 to $150 \mathrm{pmol}$ Cas9 protein in a $10 \mu \mathrm{l}$ final volume with nuclease-free water and incubating 670 at room temperature for $10 \mathrm{~min}$. On day 2 after thawing CD34+ cells, the RNP complexes 671 were added to $1 \times 10^{5}$ cells in $40 \mu$ of P3 nucleofection buffer from the 4D-Nucleofector $X$

672 kit (Lonza). Half of the mixture was loaded to each well of a 16-well nucleofection cassette 673 and nucleofected using the using E0-100 program with the 4D-Nucleofector Lonza 
674 Amaxa. After nucleofection, cells were transferred to $6 \mathrm{ml}$ fresh cPIMDM and incubated 675 at $37^{\circ} \mathrm{C}$ in $5 \% \mathrm{CO} 2$ in air.

\section{Enrichment of CD71 negative cRBCs}

678 Fully differentiated cRBCs were washed in degassed and chilled bead buffer (PBS + 0.5\%

679 bovine serum albumin $+2 \mathrm{mM}$ EDTA), resuspended in the same buffer $\left(45 \mu \mathrm{l}\right.$ for $6 \times 10^{6}$ 680 cells), and incubated the anti-CD71 antibody-immoblized beads (Miltenyi) (10 $\mu$ l beads for

$6816 \times 10^{6}$ cells) at $4^{\circ} \mathrm{C}$ for $15 \mathrm{~min}$. The cells were washed in $1 \mathrm{ml}$ of bead buffer and passed 682 through an LS magnetic column (Miltenyi) and washed 3 times with $2 \mathrm{ml}$ of the buffer. 683 The flow through containing CD71- negative cells was collected, washed with cRPMI 684 medium and used for invasion assays.

\section{Detection of cell surface proteins by flow cytometry}

687 Expression of RBC surface proteins was measured in control or knockout cRBCs by flow 688 cytometry. $1 \times 10^{6}$ cRBCs were washed 2 times with PBS/0.3\% BSA and incubated with 689 primary monoclonal antibodies or fluorochrome conjugated antibodies at $4^{\circ} \mathrm{C}$ for $1 \mathrm{~h}$. 690 Antibodies used: anti-CD55 (BRIC 216-PE, IBGRL; 1:50) and anti-CD71-PE (Miltenyi; 691 1:20). After incubation, the cells were washed 2 times in PBS/0.3\% BSA, followed by flow 692 cytometry analysis on a MACSQuant flow cytometer (Miltenyi).

694 Immunofluorecence assays (IFA)

695 IFAs were performed as previously described, with some modifications (Tonkin et al., 696 2004). For IFAs of CD55-CRISPR cRBCs infected with $P$. falciparum, cells were fixed in 
$6974.0 \%$ paraformaldehyde and $0.0015 \%$ glutaraldehyde in PBS for 20 min at room 698 temperature and blocked for one hour in 3\% BSA/PBS. The cells were incubated with 699 anti-CD55 antibody (BRIC 216-PE from IBGRL) at 1:50 concentration for $1 \mathrm{~h}$ at $4^{\circ} \mathrm{C}$. Cells 700 were mounted Fluoromount-G with DAPI mounting medium (Electron Microscopy 701 Services) and the fluorescent images were captured with a 60X objective on a Keyence 702 BZ-X700 fluorescence microscope. For IFAs of merozoites attached to pRBCs, samples 703 were prepared as in the attachment assays (see below), and 60 ul of the samples were 704 fixed in the fixative containing 4.0\% Paraformaldehyde and $0.015 \%$ Glutaraldehyde for $70520 \mathrm{~min}$, washed twice in PBS, and allowed to settle onto a Poly-L-Lysine coated coverslip 706 (Corning). The samples were then incubated in $0.1 \%$ Triton $X-100 / P B S$ for $10 \mathrm{~min}$ at 707 room temperature, washed in PBS, and incubated in $0.1 \mathrm{mg} / \mathrm{ml}$ of NaBH4/PBS for $10 \mathrm{~min}$. 708 at room temperature. Following a wash in PBS, the cells were blocked overnight in fresh 709 PBS/3.0\% BSA at room temperature. Primary antibodies: mouse monoclonal Anti710 PfAMA1 1F9 (1:200) (Coley et al., 2001) and rabbit polyclonal anti-PfRON4 (1:200)

711 (Richard et al., 2010) were diluted in blocking buffer applied to cells for $2 \mathrm{hr}$ at room 712 temperature. Following 3 washes, the cells were incubated in corresponding secondary 713 antibodies at 1:500 dilution: Alexa Fluor 555 (anti-rabbit) and Alexa Fluor 488 (anti714 mouse) for $1 \mathrm{~h}$ at room temperature. The cells were washed in PBS supplemented with $0.1 \mathrm{ng} / \mu \mathrm{l}$ of DAPI (Thermofisher) and mounted in Vectashield anti-fade mounting medium.

716 Imaging was performed using a Zeiss LSM710 confocal microscope and the image 717 analysis was performed using Fiji/imageJ (Version 2.0.0-rc-69/1.52i). Apical orientation 718 was defined as PfAMA1 and PfRON4 co-localization at the cellular interface, and non719 apical orientation was defined as PfAMA1 and PfRON4 not co-localized at the cellular 
720 interface. Merozoites were defined as being mid-invasion if AMA1 and RON4 were co-

721 localized at cellular interface and the merozoite appeared to protrude into the RBC,

722 creating an indentation.

723

724 Anti-CD55 polyclonal antibody generation

725 A CD55 cDNA (Asp 35-Ser 353) was cloned into a modified pTT5 vector (Raymond et 726 al., 2011), whose expression cassette consists of eGFP fused to a puromycin resistance 727 gene followed by a 2A skip peptide (Funston, Kallioinen, de Felipe, Ryan, \& Iggo, 2008) 728 and a BiP signal peptide, and the resulting plasmid was verified by sequencing. 1.8L of 729 293-6E cells grown in Freestyle 293 media (Invitrogen) to $0.8 \times 10$ e6 cells/ml were 730 transfected with linear PEl (Polysciences) with 397 ug DNA at a 1:3 DNA:PEI 731 ratio. TN1 tryptone (Organotechnie) was added to a final concentration of $0.5 \%(\mathrm{w} / \mathrm{v})$ 732 one day after transfection, and cells were grown for an additional 5 days. Cells were spun 733 down and discarded, and the following reagents were added to the harvested media (final 734 concentration listed): $20 \mathrm{mM}$ Tris $\mathrm{pH} 8,350 \mathrm{mM} \mathrm{NaCl}, 5 \mathrm{mM}$ Imidazole $\mathrm{pH} 8,0.2 \mathrm{mM}$ $735 \mathrm{NiCl}$. 6ml GE IMAC sepharose 6 beads, and the sample was rocked at $4 \mathrm{C}$ for 1 736 hour. The sample was then poured into a glass column, and washed with 50ml of TBS 737 (10mM Tris $\mathrm{pH} 8,500 \mathrm{mM} \mathrm{NaCl})$ with imidazole at the following concentrations 738 sequentially: $5 \mathrm{mM}, 10 \mathrm{mM}, 50 \mathrm{mM}$, and $250 \mathrm{mM}$. Nickel was stripped from the column with $73910 \mathrm{ml} 100 \mathrm{mM}$ EDTA. The majority of the CD55-8xHis protein eluted in the 250mM 740 Imidazole fraction, with a smaller amount of equal purity in the $50 \mathrm{mM}$ imadazole

741 fraction. These were dialyzed separately into PBS pH 7.4 overnight at 4C, concentrated 742 to $10 \mathrm{mg} / \mathrm{ml}$ using Amicon Ultra 15 with $30 \mathrm{kDa}$ cutoff (Millipore), snap frozen in liquid 
743 nitrogen and stored at $-80 \mathrm{C}$ until further use. $2.5 \mathrm{mg}$ of CD55-8xHis was used for three

744 immunizations of two New Zealand white-SPF rabbits by New England Peptide.

745 Approximately $50 \mathrm{ml}$ of recovered antiserum was used for negative affinity purification with

746 an 8X HIS column, and subsequently purified with a protein A column. Approximately 15

$747 \mathrm{ml}$ of purified $\mathrm{lgG}$ at $1.209 \mathrm{mg} / \mathrm{ml}$ concentration was purified and stored at $-80 \mathrm{C}$ 748 until further use.

\section{$751 \quad$ Live cell imaging}

752 Schizont stage $P$. falciparum strain 3D7 parasites at 4-5\% parasitemia and 2\% hematocrit

753 were tightly synchronized with $2 \mu \mathrm{M}$ Compound 2 to prevent schizont rupture (Collins et

754 al., 2013). After 4-6 hour incubation, they were washed three times and allowed to

755 recover in fresh cRPMI for 45 to 90 minutes at $37^{\circ} \mathrm{C}$. After recovery, anti-CD55 antibody

756 or lgG isotype control was added to the cells at final antibody concentration of $400 \mu \mathrm{g} / \mathrm{ml}$

757 and 1\% hematocrit. In experiments involving previously cryopreserved CD55-null pRBCs

758 (Takahashi, 2008) or control WT pRBCs, late stage $P$. falciparum schizonts were isolated

759 using a MACS magnet, synchronized with Compound 2, and then mixed with the pRBCs

760 at $4-5 \%$ parasitemia and $1 \%$ hematocrit. The assays were loaded into a $20 \mathrm{~mm}$ diameter

761 Hybriwell sealing system (Grace Bio-labs) customized for live cell imaging. Rupture of the

762 parasite-infected red blood cells and subsequent invasion events were video recorded

763 using a $60 \mathrm{X}$ objective Keyence BZ-X700 live microscopy setup at $37^{\circ} \mathrm{C}$ supplied with $5 \%$

$764 \mathrm{CO}_{2}$ and $1 \% \mathrm{O}_{2}$ gaseous environment. For the live cell imaging of echinocytosis, $1 \mu \mathrm{M}$

765 Cytochalasin D was added to the above mixtures. Kinetics and morphology of distinct 
766 steps of invasion in the video were measured by using ImageJ (ImageJ 1.50i) as

767 described previously(Schneider et al., 2012; Weiss et al., 2015). Briefly, the following

768 events were quantified in each video: a) Contacts between merozoites and RBCs that

769 culminated in successful invasion; b) successful invasion that resulted echinocytosis; c)

770 contacts between merozoites and RBCs that could not proceed beyond initial

771 contact/deformation. d) RBCs contacted before successful invasion e) period of pre-

772 invasion (initial contact to end of deformation), f) during of internalization, g) time to the

773 onset of echinocytosis, h) efficiency and degree of merozoite-induced deformation of the

774 target RBC using a four-point deformation scale $(0,1,2,3)$.

775

776 Merozoite attachment assays

777 Purified schizonts were added at $12.0 \%$ parasitemia to previously cryopreserved CD55-

778 null pRBCs (Takahashi, 2008) or control WT pRBCs at 1.0\% hematocrit in the presence

779 of $1 \mu \mathrm{M}$ Cytochalasin D, $50 \mathrm{U} / \mathrm{ml}$ Heparin or none in a final volume of $100 \mathrm{ul}$ per well in a

78096 well plate. The mixtures were incubated at $37^{\circ} \mathrm{C}$ for $90 \mathrm{~min}$ and the schizonts were

781 allowed to rupture. To quantify merozoite attachment by flow cytometry, $10 \mu$ liquots of

782 the samples were fixed in $2 \%$ Glutaraldehyde and $0.116 \mathrm{M}$ sucrose in PBS, washed in

783 PBS/0.3\% BSA, and stained with SYBR Green 1 nucleic acid stain (Invitrogen). at 1:2000

784 dilution in PBS/0.3\% BSA for 20 minutes, followed by flow cytometry analysis on a

785 MACSQuant flow cytometer (Miltenyi). The assays were performed 3 times with 3

786 technical replicates. The merozoite attachment rates to control or CD55-null RBCs were

787 calculated as the percent of RBCs with an attached merozoite, and were normalized for

788 background attachment in the presence of heparin. The merozoite attachment efficiency 
bioRxiv preprint doi: https://doi.org/10.1101/2020.07.27.223644; this version posted July 28, 2020. The copyright holder for this preprint (which

was not certified by peer review) is the author/funder, who has granted bioRxiv a license to display the preprint in perpetuity. It is made available under aCC-BY-NC-ND 4.0 International license.

789 was calculated for each genetic background in each biological replicate by normalizing to

790 the mean of the attachment rates in the three control experiments.

791

\section{Data analysis}

793 Stastistical analyses were performed and the graphs were generated using GraphPad Prism

7948 Version 8.0.2 (159) for macOS.

795

796 


\section{Supplemental Information}

798 Supplemental information includes six figures and eight movies.

799

800 Multimedia Files

801 Movie S1: P. falciparum 3D7 invasion in the presence of isotype control antibody.

802 Movie S2: P. falciparum 3D7 invasion in the presence of anti-CD55 antibody.

803 Movie S3: P. falciparum 3D7 merozoite-induced deformation with deformation score 1.

804 Movie S4: P. falciparum 3D7 merozoite-induced deformation with deformation score 2.

805 Movie S5: P. falciparum 3D7 merozoite-induced deformation with deformation score 3.

806 Movie S6: An example of a failed invasion despite successful merozoite-induced

807 deformation in CD55-null pRBC.

808 Movie S7: An example of a successful invasion of a wild-type pRBC.

809 Movie S8: Echinocytosis induced by attached $P$. falciparum merozoite to pRBCs in the

810 presence of Cytochalasin D. Black arrows: Attachment resulting echinocyte formation.

811 White arrows: Attachment resulting no echinocyte formation. 


\section{References}

Alexander, D. L., Arastu-Kapur, S., Dubremetz, J. F., \& Boothroyd, J. C. (2006). Plasmodium falciparum AMA1 binds a rhoptry neck protein homologous to TgRON4, a component of the moving junction in Toxoplasma gondii. Eukaryot Cell, 5(7), 1169-1173. doi:10.1128/EC.00040-06

Alexander, D. L., Mital, J., Ward, G. E., Bradley, P., \& Boothroyd, J. C. (2005). Identification of the moving junction complex of Toxoplasma gondii: a collaboration between distinct secretory organelles. PLoS Pathog, 1(2), e17. doi:10.1371/journal.ppat.0010017

Bei, A. K., \& Duraisingh, M. T. (2012). Functional analysis of erythrocyte determinants of Plasmodium infection. Int J Parasitol, 42(6), 575-582. doi:10.1016/j.ijpara.2012.03.006

Besteiro, S., Dubremetz, J. F., \& Lebrun, M. (2011). The moving junction of apicomplexan parasites: a key structure for invasion. Cell Microbiol, 13(6), 797-805. doi:10.1111/j.1462-5822.2011.01597.x

Besteiro, S., Michelin, A., Poncet, J., Dubremetz, J. F., \& Lebrun, M. (2009). Export of a Toxoplasma gondii rhoptry neck protein complex at the host cell membrane to form the moving junction during invasion. PLoS Pathog, 5(2), e1000309. doi:10.1371/journal.ppat.1000309

Chen, L., Lopaticki, S., Riglar, D. T., Dekiwadia, C., Uboldi, A. D., Tham, W. H., . . Cowman, A. F. (2011). An EGF-like protein forms a complex with PfRh5 and is required for invasion of human erythrocytes by Plasmodium falciparum. PLoS Pathog, 7(9), e1002199. doi:10.1371/journal.ppat.1002199

Chu, T. T. T., Sinha, A., Malleret, B., Suwanarusk, R., Park, J. E., Naidu, R., . . . Chandramohanadas, R. (2018). Quantitative mass spectrometry of human reticulocytes reveal proteome-wide modifications during maturation. Br J Haematol, 180(1), 118-133. doi:10.1111/bjh.14976

Coley, A. M., Campanale, N. V., Casey, J. L., Hodder, A. N., Crewther, P. E., Anders, R. F., .. . Foley, M. (2001). Rapid and precise epitope mapping of monoclonal antibodies against Plasmodium falciparum AMA1 by combined phage display of fragments and random peptides. Protein Eng, 14(9), 691-698. doi:10.1093/protein/14.9.691

Collins, C. R., Hackett, F., Strath, M., Penzo, M., Withers-Martinez, C., Baker, D. A., \& Blackman, M. J. (2013). Malaria parasite cGMP-dependent protein kinase regulates blood stage merozoite secretory organelle discharge and egress. PLoS Pathog, 9(5), e1003344. doi:10.1371/journal.ppat.1003344 
Cooling, L. (2015). Blood Groups in Infection and Host Susceptibility. Clin Microbiol Rev, 28(3), 801-870. doi:10.1128/CMR.00109-14

Coyne, C. B., \& Bergelson, J. M. (2006). Virus-induced Abl and Fyn kinase signals permit coxsackievirus entry through epithelial tight junctions. Cell, 124(1), 119-131. doi:10.1016/j.cell.2005.10.035

Crosnier, C., Bustamante, L. Y., Bartholdson, S. J., Bei, A. K., Theron, M., Uchikawa, M., . . . Wright, G. J. (2012). Basigin is a receptor essential for erythrocyte invasion by Plasmodium falciparum. Nature, 480(7378), 534-537. doi:nature10606 [pii] $\underline{10.1038 / \text { nature } 10606}$

Dreyer, A. M., Matile, H., Papastogiannidis, P., Kamber, J., Favuzza, P., Voss, T. S., . . Pluschke, G. (2012). Passive immunoprotection of Plasmodium falciparum-infected mice designates the CyRPA as candidate malaria vaccine antigen. J Immunol, 188(12), 62256237. doi:10.4049/jimmunol.1103177

Dvorak, J. A., Miller, L. H., Whitehouse, W. C., \& Shiroishi, T. (1975). Invasion of erythrocytes by malaria merozoites. Science, 187(4178), 748-750. doi:10.1126/science.803712

Egan, E. S. (2017). Beyond Hemoglobin: Screening for Malaria Host Factors. Trends Genet. doi:10.1016/j.tig.2017.11.004

Egan, E. S., Jiang, R. H., Moechtar, M. A., Barteneva, N. S., Weekes, M. P., Nobre, L. V., . . . Duraisingh, M. T. (2015). Malaria. A forward genetic screen identifies erythrocyte CD55 as essential for Plasmodium falciparum invasion. Science, 348(6235), 711-714. doi:10.1126/science.aaa3526

Funston, G. M., Kallioinen, S. E., de Felipe, P., Ryan, M. D., \& Iggo, R. D. (2008). Expression of heterologous genes in oncolytic adenoviruses using picornaviral $2 \mathrm{~A}$ sequences that trigger ribosome skipping. J Gen Virol, 89(Pt 2), 389-396. doi:10.1099/vir.0.83444-0

Gautier, E. F., Ducamp, S., Leduc, M., Salnot, V., Guillonneau, F., Dussiot, M., . . Mayeux, P. (2016). Comprehensive Proteomic Analysis of Human Erythropoiesis. Cell Rep, 16(5), 1470-1484. doi:10.1016/j.celrep.2016.06.085

Giarratana, M. C., Kobari, L., Lapillonne, H., Chalmers, D., Kiger, L., Cynober, T., . . Douay, L. (2005). Ex vivo generation of fully mature human red blood cells from hematopoietic stem cells. Nat Biotechnol, 23(1), 69-74. doi:nbt1047 [pii]10.1038/nbt1047

Giarratana, M. C., Rouard, H., Dumont, A., Kiger, L., Safeukui, I., Le Pennec, P. Y., . . Douay, L. (2011). Proof of principle for transfusion of in vitro-generated red blood cells. Blood, 118(19), 5071-5079. doi:blood-2011-06-362038 [pii] 10.1182/blood-2011-06-362038 
Gilson, P. R., \& Crabb, B. S. (2009). Morphology and kinetics of the three distinct phases of red blood cell invasion by Plasmodium falciparum merozoites. Int J Parasitol, 39(1), 91-96. doi:10.1016/j.ijpara.2008.09.007

Harvey, K. L., Yap, A., Gilson, P. R., Cowman, A. F., \& Crabb, B. S. (2014). Insights and controversies into the role of the key apicomplexan invasion ligand, Apical Membrane Antigen 1. Int J Parasitol, 44(12), 853-857. doi:10.1016/j.ijpara.2014.08.001

Hendel, A., Bak, R. O., Clark, J. T., Kennedy, A. B., Ryan, D. E., Roy, S., . . Porteus, M. H. (2015). Chemically modified guide RNAs enhance CRISPR-Cas genome editing in human primary cells. Nat Biotechnol, 33(9), 985-989. doi:10.1038/nbt.3290

Hu, G., Cabrera, A., Kono, M., Mok, S., Chaal, B. K., Haase, S., . . Bozdech, Z. Transcriptional profiling of growth perturbations of the human malaria parasite Plasmodium falciparum. Nat Biotechnol, 28(1), 91-98. doi:nbt.1597 [pii] 10.1038/nbt.1597

Hu, J., Liu, J., Xue, F., Halverson, G., Reid, M., Guo, A., . . An, X. (2013). Isolation and functional characterization of human erythroblasts at distinct stages: implications for understanding of normal and disordered erythropoiesis in vivo. Blood, 121(16), 32463253. doi:10.1182/blood-2013-01-476390

Kanjee, U., Gruring, C., Chaand, M., Lin, K. M., Egan, E., Manzo, J., .. . Duraisingh, M. T. (2017). CRISPR/Cas9 knockouts reveal genetic interaction between strain-transcendent erythrocyte determinants of Plasmodium falciparum invasion. Proc Natl Acad Sci U S A, 114(44), E9356-E9365. doi:10.1073/pnas.1711310114

Koch, M., \& Baum, J. (2016). The mechanics of malaria parasite invasion of the human erythrocyte - towards a reassessment of the host cell contribution. Cell Microbiol, 18(3), 319-329. doi:10.1111/cmi.12557

Malleret, B., Xu, F., Mohandas, N., Suwanarusk, R., Chu, C., Leite, J. A., . . Russell, B. (2013). Significant biochemical, biophysical and metabolic diversity in circulating human cord blood reticulocytes. PLoS One, 8(10), e76062. doi:10.1371/journal.pone.0076062

Miller, L. H., Aikawa, M., Johnson, J. G., \& Shiroishi, T. (1979). Interaction between cytochalasin B-treated malarial parasites and erythrocytes. Attachment and junction formation. $J$ Exp Med, 149(1), 172-184.

Ord, R. L., Rodriguez, M., \& Lobo, C. A. (2015). Malaria invasion ligand RH5 and its prime candidacy in blood-stage malaria vaccine design. Hum Vaccin Immunother, 11(6), 14651473. doi:10.1080/21645515.2015.1026496 
Pasvol, G., Chasis, J. A., Mohandas, N., Anstee, D. J., Tanner, M. J., \& Merry, A. H. (1989). Inhibition of malarial parasite invasion by monoclonal antibodies against glycophorin $A$ correlates with reduction in red cell membrane deformability. Blood, 74(5), 1836-1843.

Paul, A. S., Egan, E. S., \& Duraisingh, M. T. (2015). Host-parasite interactions that guide red blood cell invasion by malaria parasites. Curr Opin Hematol, 22(3), 220-226. doi:10.1097/MOH.0000000000000135

Raymond, C., Tom, R., Perret, S., Moussouami, P., L'Abbe, D., St-Laurent, G., \& Durocher, Y. (2011). A simplified polyethylenimine-mediated transfection process for large-scale and high-throughput applications. Methods, 55(1), 44-51. doi:10.1016/j.ymeth.2011.04.002

Reddy, K. S., Amlabu, E., Pandey, A. K., Mitra, P., Chauhan, V. S., \& Gaur, D. (2015). Multiprotein complex between the GPI-anchored CyRPA with PfRH5 and PfRipr is crucial for Plasmodium falciparum erythrocyte invasion. Proc Natl Acad Sci U S A, 112(4), 11791184. doi:10.1073/pnas.1415466112

Richard, D., MacRaild, C. A., Riglar, D. T., Chan, J. A., Foley, M., Baum, J., . . Cowman, A. F. (2010). Interaction between Plasmodium falciparum apical membrane antigen 1 and the rhoptry neck protein complex defines a key step in the erythrocyte invasion process of malaria parasites. J Biol Chem, 285(19), 14815-14822. doi:10.1074/jbc.M109.080770

Riglar, D. T., Richard, D., Wilson, D. W., Boyle, M. J., Dekiwadia, C., Turnbull, L., . . Baum, J. (2011). Super-resolution dissection of coordinated events during malaria parasite invasion of the human erythrocyte. Cell Host Microbe, 9(1), 9-20. doi:10.1016/j.chom.2010.12.003

Sack, B., Kappe, S. H., \& Sather, D. N. (2017). Towards functional antibody-based vaccines to prevent pre-erythrocytic malaria infection. Expert Rev Vaccines, 16(5), 403-414. doi:10.1080/14760584.2017.1295853

Salinas, N. D., Tang, W. K., \& Tolia, N. H. (2019). Blood-Stage Malaria Parasite Antigens: Structure, Function, and Vaccine Potential. J Mol Biol, 431(21), 4259-4280. doi:10.1016/j.jmb.2019.05.018

Satchwell, T. J., Wright, K. E., Haydn-Smith, K. L., Sanchez-Roman Teran, F., Moura, P. L., Hawksworth, J., . . . Baum, J. (2019). Genetic manipulation of cell line derived reticulocytes enables dissection of host malaria invasion requirements. Nat Commun, 10(1), 3806. doi:10.1038/s41467-019-11790-w

Schneider, C. A., Rasband, W. S., \& Eliceiri, K. W. (2012). NIH Image to ImageJ: 25 years of Image Analysis. Nature Methods, 9(7), 671-675. 
Scully, E. J., Shabani, E., Rangel, G. W., Gruring, C., Kanjee, U., Clark, M. A., . . Duraisingh, M. T. (2019). Generation of an immortalized erythroid progenitor cell line from peripheral blood: A model system for the functional analysis of Plasmodium spp. invasion. Am J Hematol, 94(9), 963-974. doi:10.1002/ajh.25543

Spadafora, C., Awandare, G. A., Kopydlowski, K. M., Czege, J., Moch, J. K., Finberg, R. W., . . . Stoute, J. A. (2010). Complement receptor 1 is a sialic acid-independent erythrocyte receptor of Plasmodium falciparum. PLoS Pathog, 6(6), e1000968. doi:10.1371/journal.ppat.1000968

Srinivasan, P., Beatty, W. L., Diouf, A., Herrera, R., Ambroggio, X., Moch, J. K., . . Miller, L. H. (2011). Binding of Plasmodium merozoite proteins RON2 and AMA1 triggers commitment to invasion. Proc Natl Acad Sci U S A, 108(32), 13275-13280. doi:10.1073/pnas.1110303108

Storry, J. R., Reid, M. E., \& Yazer, M. H. (2010). The Cromer blood group system: a review. Immunohematology, 26(3), 109-118.

Takahashi, J. Y. S., Yamane S, Tanaka M, Takahashi H, et al. (2008). A case of Inab phenotype (IFC-) with anti-IFC. Jpn J Transfusion and Cell Therapy, 54(2), 258.

Taylor, S. M., \& Fairhurst, R. M. (2014). Malaria parasites and red cell variants: when a house is not a home. Curr Opin Hematol, 21(3), 193-200. doi:10.1097/MOH.0000000000000039

Tham, W. H., Healer, J., \& Cowman, A. F. (2012). Erythrocyte and reticulocyte binding-like proteins of Plasmodium falciparum. Trends Parasitol, 28(1), 23-30. doi:10.1016/j.pt.2011.10.002

Tiffert, T., Lew, V. L., Ginsburg, H., Krugliak, M., Croisille, L., \& Mohandas, N. (2005). The hydration state of human red blood cells and their susceptibility to invasion by Plasmodium falciparum. Blood, 105(12), 4853-4860. doi:10.1182/blood-2004-12-4948

Tonkin, C. J., van Dooren, G. G., Spurck, T. P., Struck, N. S., Good, R. T., Handman, E., . . . McFadden, G. I. (2004). Localization of organellar proteins in Plasmodium falciparum using a novel set of transfection vectors and a new immunofluorescence fixation method. Mol Biochem Parasitol, 137(1), 13-21. doi:10.1016/j.molbiopara.2004.05.009

Volz, J. C., Yap, A., Sisquella, X., Thompson, J. K., Lim, N. T., Whitehead, L. W., . . Cowman, A. F. (2016). Essential Role of the PfRh5/PfRipr/CyRPA Complex during Plasmodium falciparum Invasion of Erythrocytes. Cell Host Microbe, 20(1), 60-71. doi:10.1016/j.chom.2016.06.004

Weiss, G. E., Gilson, P. R., Taechalertpaisarn, T., Tham, W. H., de Jong, N. W., Harvey, K. L., . . . Crabb, B. S. (2015). Revealing the Sequence and Resulting Cellular Morphology of 
Receptor-Ligand Interactions during Plasmodium falciparum Invasion of Erythrocytes.

1033

1034

PLoS Pathog, 11(2), e1004670. doi:10.1371/journal.ppat.1004670

1035

1036

WHO. (2018). World Malaria Report 2018. World Health Organization, Geneva.

1037

Yap, A., Azevedo, M. F., Gilson, P. R., Weiss, G. E., O'Neill, M. T., Wilson, D. W., ... Cowman, A. F. (2014). Conditional expression of apical membrane antigen 1 in Plasmodium

1039 falciparum shows it is required for erythrocyte invasion by merozoites. Cell Microbiol, 16(5), 642-656. doi:10.1111/cmi.12287 